\title{
TROPICAL COMBINATORICS AND WHITTAKER FUNCTIONS
}

\author{
IVAN CORWIN, NEIL O'CONNELL, TIMO SEPPÄLÄINEN, AND NIKOLAOS ZYGOURAS
}

\begin{abstract}
We establish a fundamental connection between the geometric RSK correspondence and $G L(N, \mathbb{R})$ Whittaker functions, analogous to the well known relationship between the RSK correspondence and Schur functions. This gives rise to a natural family of measures associated with $G L(N, \mathbb{R})$-Whittaker functions which are the analogues in this setting of the Schur measures on integer partitions. The corresponding analogue of the Cauchy-Littlewood identity can be seen as a generalisation of an integral identity for $G L(N, \mathbb{R})$ Whittaker functions due to Bump and Stade. As an application, we obtain an explicit integral formula for the Laplace transform of the law of the partition function associated with a one-dimensional directed polymer model with log-gamma weights recently introduced by one of the authors (TS).
\end{abstract}

\section{INTRODUCTION}

The Robinson-Schensted-Knuth (RSK) correspondence is a combinatorial mapping which plays a fundamental role in the theory of Young tableaux, symmetric functions and representation theory. In particular, it provides a direct combinatorial proof of the Cauchy-Littlewood identity

$$
\sum_{\lambda} s_{\lambda}(x) s_{\lambda}(y)=\prod_{i, j}\left(1-x_{i} y_{j}\right)^{-1}
$$

where the sum is over integer partitions and $s_{\lambda}$ denotes the Schur function associated with the partition $\lambda$. The Schur function $s_{\lambda}(x)$ is a symmetric function in the variables $x=\left(x_{1}, x_{2}, \ldots\right)$ defined by

$$
s_{\lambda}(x)=\sum_{T} x^{T}
$$

where the sum is over semistandard tableaux $T$ with shape $\lambda$ and $x^{T}=x_{1}^{\mu_{1}} x_{2}^{\mu_{2}} \ldots$, where $\mu_{i}$ is the number of $i$ 's in $T$. For more background on symmetric functions we refer the reader to [40].

The RSK mapping is defined by a combinatorial algorithm which associates to each matrix $M=\left\{m_{i j}\right\}$ with non-negative integer entries a pair $(P, Q)$ of semi-standard tableaux with the same shape. By a version of Greene's theorem [32, 36], it can also be defined via expressions in the $(\max ,+)$ semi-ring. This was extended to matrices with real entries by Berenstein and Kirillov [9]. Replacing these expressions by their analogues in the usual $(+, \times)$ algebra, A.N. Kirillov [36] introduced a geometric lifting of the BerensteinKirillov correspondence which he called the 'tropical RSK correspondence', in honour of M.-P. Schützenberger (1920-1996). However, for many readers nowadays the word 'tropical' indicates just the opposite, so to avoid confusion we will refer to Kirillov's construction as the geometric RSK correspondence, as in the theory of geometric crystals $[7,8]$, which is closely related. This correspondence has been studied further from a dynamical point of view by Noumi and Yamada [43] and related to Dodgson's condensation method for computing determinants in [21].

It is natural to ask if there is an analogue of the Cauchy-Littlewood identity for the geometric RSK correspondence. A priori it is not at all clear that this should exist: something quite remarkable needs to happen in order to see the product structures on both sides which characterise this identity. In this paper we show that in fact these product structures do appear in the correct formulation and, moreover, the role of the Schur functions is now played by $G L(N, \mathbb{R})$-Whittaker functions. In a particular case (corresponding to square matrices) the analogue of the Cauchy-Littlewood identity is in fact a well known integral identity for $G L(N, \mathbb{R})$-Whittaker functions due to Bump and Stade [17, 61, 29].

Before stating our main results, we will first explain the origin of these product structures in the context of the RSK correspondence and its role in the proof of the Cauchy-Littlewood identity. The RSK mapping

Date: May 15, 2013. 
associates to each matrix $M=\left\{m_{i j}\right\}$ with non-negative integer entries a pair $(P, Q)$ of semi-standard tableaux with the same shape. If $M$ is an $n \times N$ matrix then $P$ has entries from $\{1,2, \ldots, N\}$ and $Q$ has entries from $\{1,2, \ldots, n\}$. Moreover, the vector $\left(R_{1}, \ldots, R_{n}\right)$ of row sums of $M$ is the type of $P$, that is, $R_{1}$ is the number of 1's in the semistandard tableau $P, R_{2}$ is the number of 2's, and so on. Similarly, the vector of column sums $\left(C_{1}, \ldots, C_{N}\right)$ is the type of $Q$. Note that this reflects the well known symmetry property of RSK, namely that if $M \mapsto(P, Q)$ then $M^{t} \mapsto(Q, P)$. It follows that

$$
\prod_{i, j}\left(x_{i} y_{j}\right)^{m_{i j}}=\prod_{i} x^{R_{i}} \prod_{j} y^{C_{j}}=x^{P} y^{Q}
$$

and summing both sides gives the Cauchy-Littlewood identity: on the left we sum over all matrices with non-negative integer entries and on the right we sum over all pairs of semistandard tableaux $P$ and $Q$ with the same shape.

Another way of interpreting the above argument is as follows. Let $p_{1}, \ldots, p_{n}$ and $q_{1}, \ldots, q_{N}$ be a collection of positive numbers such that $0<p_{i} q_{j}<1$ for all $i$ and $j$, and consider the probability measure on $n \times N$ matrices defined by

$$
P(\{M\})=\prod_{i, j}\left(1-p_{i} q_{j}\right) \prod_{i, j}\left(p_{i} q_{j}\right)^{m_{i j}} .
$$

From the above discussion, the push-forward of this probability measure onto the shape of the tableaux obtained under the RSK mapping is given by

$$
\tilde{P}(\{\lambda\})=\prod_{i, j}\left(1-p_{i} q_{j}\right) s_{\lambda}(p) s_{\lambda}(q) .
$$

Now, the Cauchy-Littlewood identity is essentially equivalent to the fact that $\tilde{P}$ is a probability measure on the set of integer partitions. Such probability measures are known as Schur measures [52].

We now turn to the geometric RSK correspondence. The input is an $n \times N$ matrix $X=\left\{x_{i j}\right\}$ with strictly positive real entries and, supposing here for convenience that $n \geq N$, the analogue of the $P$-tableau is a triangular array $\left(z_{k, \ell}\right)_{1 \leq \ell \leq k \leq N}$ of non-negative real numbers. The 'shape' of $P$ is the vector $z_{N, . .}$ Let $\hat{\theta}_{1}, \ldots, \hat{\theta}_{n}$ and $\theta_{1}, \ldots, \theta_{N}$ be a collection of real numbers satisfying $\hat{\theta}_{i}+\theta_{j}>0$ for all $i$ and $j$, and consider the product measure on input matrices $X$ defined by

$$
\mu(d X)=\prod_{i, j} \nu_{\hat{\theta}_{i}+\theta_{j}}\left(d x_{i j}\right),
$$

where $\nu_{\theta}$ denotes the distribution of the inverse of a Gamma random variable

$$
\nu_{\theta}(d x)=\frac{1}{\Gamma(\theta)} x^{-\theta-1} \exp \left\{-\frac{1}{x}\right\} d x .
$$

The main result of this paper (Theorem 3.9) is that the push-forward of the probability measure $\mu$ under the geometric RSK mapping onto the 'shape' $\left(z_{N, \ell}\right)_{1 \leq \ell \leq N}$ is given by

$$
\mu_{n}^{N}(d y)=\Psi_{\theta}(y) \int_{\iota \mathbb{R}^{N}} d \lambda s_{N}(\lambda) \Psi_{-\lambda}(y) \prod_{m=1}^{n} \prod_{i=1}^{N} \frac{\Gamma\left(\hat{\theta}_{m}+\lambda_{i}\right)}{\Gamma\left(\theta_{i}+\hat{\theta}_{m}\right)} \prod_{i=1}^{N} \frac{d y_{i}}{y_{i}},
$$

where the functions $\Psi_{\nu}(y)$ are $G L(N, \mathbb{R})$-Whittaker functions (defined in Section 3.2 below) and

$$
s_{N}(\lambda)=\frac{1}{(2 \pi \iota)^{N} N !} \prod_{j \neq k} \Gamma\left(\lambda_{j}-\lambda_{k}\right)^{-1} .
$$

The probability measure $\mu_{n}^{N}$ is the analogue of the Schur measure in this setting and shall be referred to as a Whittaker measure; the fact that it integrates to one is the analogue of the Cauchy-Littlewood identity.

In the special case $N=n$, we obtain the following simplification:

$$
\mu_{N}^{N}(d y)=\prod_{m=1}^{N} \prod_{i=1}^{N} \Gamma\left(\theta_{i}+\hat{\theta}_{m}\right)^{-1} e^{-y_{N}^{-1}} \Psi_{\theta}(y) \Psi_{\hat{\theta}}(y) \prod_{i=1}^{N} \frac{d y_{i}}{y_{i}} .
$$


In this case, the analogue of the Cauchy-Littlewood identity reduces to

$$
\int_{\mathbb{R}_{+}^{N}} e^{-y_{N}^{-1}} \Psi_{\theta}(y) \Psi_{\hat{\theta}}(y) \prod_{i=1}^{N} \frac{d y_{i}}{y_{i}}=\prod_{m=1}^{N} \prod_{i=1}^{N} \Gamma\left(\theta_{i}+\hat{\theta}_{m}\right),
$$

which can be seen to be equivalent to a Whittaker integral identity due to Bump and Stade [17, 61, 29]. We remark that, while this gives a new interpretation of the Bump-Stade Whittaker integral identity, we do not give a new proof of this identity in the present paper; in fact, we use it to obtain the simplification (1.3).

We prove Theorem 3.9 by considering a dynamical version of the geometric RSK construction due to Noumi and Yamada [43], allowing $n$ to increase as we successively add rows to the input matrix $X$. The image triangular array $z(n)$ evolves as a Markov process in discrete time $n$ subject to a particular entrance law for $n<N$. First we prove that the shape $z_{N, \cdot} \cdot(n)$ evolves marginally as a Markov process in its own filtration. The Markov property of $z_{N, .}(n)$ relies (via the theory of Markov functions) on an algebraic intertwining relation between Markov kernels for $z(n)$ and $z_{N, .} \cdot(n)$ as well as on a limiting argument which shows that the result holds for the particular entrance law for $z(n)$ dictated by the geometric RSK correspondence. Secondly we prove that this Markov process can be diagonalized in terms of $G L(N, \mathbb{R})$-Whittaker functions. This yields the formula (1.2) for the fixed $n$ probability distribution of the shape.

Our results have an important application to the study of directed polymers, analogous to the role of the RSK correspondence in the study of longest increasing subsequences and last passage percolation [2, 3, 33, $34,39,63]$. According to the definition of geometric RSK, we can write $z_{N, 1}(n)=\sum_{\pi} \prod_{(i, j) \in \pi} x_{i j}$ where the summation is over all 'up/right' lattice paths in $\mathbb{Z}^{2}$ from $(1,1)$ to $(n, N)$. Under the measure $\mu$, this random variable can be interpreted as a partition function for a directed polymer in a random environment given by the weights $x_{i j}$. For a particular (homogeneous) choice of the parameters $\hat{\theta}_{i}+\theta_{j}=\gamma$ for all $i$ and $j$, this model was introduced and studied in the paper [59]. In particular, setting $Z_{n}=z_{n, 1}(n)$, it was shown in [59] that the free energy is given explicitly by

$$
\lim _{n \rightarrow \infty} \frac{1}{n} \log Z_{n}=-2 \Psi(\gamma / 2)
$$

almost surely, where $\Psi(x)=[\log \Gamma]^{\prime}(x)$ is the digamma function, and moreover

$$
\limsup _{n \rightarrow \infty} \frac{\operatorname{var} \log Z_{n}}{n^{2 / 3}}<\infty
$$

These asymptotic results were obtained via the observation that for this polymer model there is an analogue of the output theorem (or Burke property) for the single server queue. This observation, combined with recent progress on a related model in [44], provided the inspiration for the present work. In fact, the model considered in [44] is a degeneration of the one we presently consider (c.f. Section 4.2).

Using our main result, we obtain the following explicit formula for the Laplace transform of the law of the partition function $z_{N, 1}(n)$ under the measure $\mu$ (this statement is also contained in Theorem 3.9):

$$
\mathbb{E}\left[e^{-s z_{N, 1}(n)}\right]=\int_{\iota \mathbb{R}^{N}} d \lambda s^{\sum_{i=1}^{N}\left(\theta_{i}-\lambda_{i}\right)} \prod_{1 \leq i, j \leq N} \Gamma\left(\lambda_{i}-\theta_{j}\right) \prod_{m=1}^{n} \prod_{i=1}^{N} \frac{\Gamma\left(\lambda_{i}+\hat{\theta}_{m}\right)}{\Gamma\left(\theta_{i}+\hat{\theta}_{m}\right)} s_{N}(\lambda),
$$

where the poles of the functions $\Gamma\left(\lambda_{i}-\theta_{j}\right)$ and $\Gamma\left(\lambda_{i}+\hat{\theta}_{m}\right)$ are not encountered as we may assume without loss of generality that $\hat{\theta}_{m}>0$ for all $m$ and $\theta_{j}<0$ for all $j$.

Our formula (1.6) has recently been applied in [13] to prove the following asymptotic result: there exists $\gamma^{*}>0$ such that the inverse-gamma weight polymer free energy with parameter $\gamma \in\left(0, \gamma^{*}\right)$ has limiting fluctuation distribution given by

$$
\lim _{n \rightarrow \infty} \mathbb{P}\left(\frac{\log Z_{n}-n \bar{f}_{\gamma}}{n^{1 / 3}} \leq r\right)=F_{\mathrm{GUE}}\left(\left(\frac{\bar{g}_{\gamma}}{2}\right)^{-1 / 3} r\right)
$$

where $\bar{f}_{\gamma}=-2 \Psi(\gamma / 2), \bar{g}_{\gamma}=-2 \Psi^{\prime \prime}(\gamma / 2)$ and $F_{\mathrm{GUE}}$ is the GUE Tracy-Widom distribution function. The restriction on the parameter $\gamma$ is present for purely technical reasons.

There have been many recent developments on the Kardar-Parisi-Zhang (KPZ) equation [19, 26, 55] and various discretisations of this equation which have an underlying algebraic structure. The latter include the Whittaker measures and processes introduced in the present paper, and a particular family of degenerations 
of these which were introduced earlier in [44]. These in turn can be seen as degenerations of the Macdonald measures and processes introduced in [12] which gives access to the theory of Macdonald polynomials and incorporates a wide range of other interesting degenerations including $q$-deformations of the Whittaker measures and processes discussed here. A feature which is important for the Whittaker measures we consider here is the fundamental link to the geometric RSK correspondence. In particular, our main result is not a degeneration of any of the main results in the more recent paper [12].

Further developments appear in the recent work [48] which explores this connection from a combinatorial point of view, in contrast to the dynamical approach of the present work. The paper [48] provides further insight into the appearance of Whittaker functions in this setting and, among other things, studies the restriction of the geometric RSK mapping to symmetric matrices.

In another direction, it is possible to define an analogue of the geometric RSK mapping directly in the continuum setting of the KPZ equation, with input given by a two-dimensional strip of space-time white noise [49]. Some recent progress towards understanding the law of the analogue of the 'shape' in this setting has been made in $[12,20]$.

The outline of the paper is as follows. In Section 2 we introduce the geometric RSK correspondence via two equivalent approaches: row insertion procedure and non-intersecting lattice paths. Section 3 provides the main set of results of this paper. We state the main algebraic content of the paper in the form of the intertwining relation of Proposition 3.4; we define Whittaker functions; we state the paper's main results Theorems 3.7 and 3.9). Within that section we also record the invariant distribution of dynamical geometric RSK correspondence, and explain the connection Pitman's $2 M-X$ theorem. Section 4 details how in certain scaling limits of our work, one recovers previously discovered results. Proofs of our main results are contained in Section 5.

Acknowledgements. We thank Jinho Baik, Gerard Ben Arous, Philippe Biane, Alexei Borodin, Percy Deift, Jeremy Quastel, Pierre van Moerbeke, Herbert Spohn, Craig Tracy and Lauren Williams for helpful discussions, and acknowledge MSRI, IMPA, Mathematisches Forschungsinstitut Oberwolfach and the University of Warwick (with financial support from grants EP/I014829/1 and IRG-246809) for hospitality during this project. IC is partially supported by the NSF through PIRE grant OISE-07-30136 and DMS-1208998 as well as by Microsoft Research through the Schramm Memorial Fellowship, and by the Clay Mathematics Institute through a Clay Research Fellowship. NO'C is partially supported by EPSRC grant EP/I014829/1. TS is partially supported by National Science Foundation grant DMS-1003651 and by the Wisconsin Alumni Research Foundation. NZ is partially supported by a Marie Curie International Reintegration Grant within the 7th European Community Framework Programme, IRG-246809.

\section{Geometric RSK correspondence}

In this section we describe an extension of Kirillov's 'tropical' (or presently 'geometric') RSK correspondence [36] to rectangular matrices. We follow mainly the development in [43] but with a slightly different convention for indices. We describe first a geometric row insertion procedure, and then expand this into a procedure for inserting a word into a triangular array (see Example 2.3 for a step-by-step illustration of these procedures). Repeated insertions create a temporal evolution of the array. In addition to insertion into an already existing array we consider insertion into an initially empty array. This latter version will have an equivalent description in terms of weights of configurations of lattice paths.

\subsection{Geometric RSK via row insertion.}

Definition 2.1. Let $1 \leq \ell \leq N$. Consider two words $\xi=\left(\xi_{\ell}, \ldots, \xi_{N}\right)$ and $b=\left(b_{\ell}, \ldots, b_{N}\right)$ with strictly positive real entries. Geometric row insertion of the word $b$ into the word $\xi$ transforms $(\xi, b)$ into a new pair $\left(\xi^{\prime}, b^{\prime}\right)$ where $\xi^{\prime}=\left(\xi_{\ell}^{\prime}, \ldots, \xi_{N}^{\prime}\right)$ and $b^{\prime}=\left(b_{\ell+1}^{\prime}, \ldots, b_{N}^{\prime}\right)$. The transformation is notated and defined as follows:

$$
\xi \underset{b^{\prime}}{\underset{b}{\downarrow}} \quad \xi^{\prime} \quad \text { where } \begin{cases}\xi_{\ell}^{\prime}=b_{\ell} \xi_{\ell}, \\ \xi_{k}^{\prime}=b_{k}\left(\xi_{k-1}^{\prime}+\xi_{k}\right), & \ell+1 \leq k \leq N \\ b_{k}^{\prime}=b_{k} \frac{\xi_{k} \xi_{k-1}^{\prime}}{\xi_{k-1} \xi_{k}^{\prime}}, & \ell+1 \leq k \leq N .\end{cases}
$$




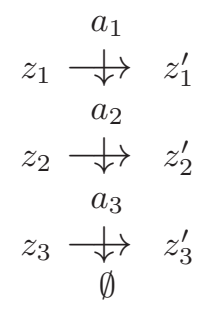

FiguRE 1. Illustration of $z^{\prime}=z \leftarrow a_{1}$ when $N=3$. Geometric row insertion of the word $a_{1}=\left(a_{11}, a_{21}, a_{31}\right)$ into the triangular array $z$ is defined recursively by insertion of $a_{i}$ into $z_{i}$ with outputs $z_{i}^{\prime}$ and $a_{i+1}$. After step 3 the process has been exhausted: $a_{3}=\left(a_{33}\right)$ has one entry and $a_{4}$ is an empty vector.

If $\ell=N$ output $b^{\prime}$ is empty and we write $b^{\prime}=\emptyset$. In addition to $\xi \in(0, \infty)^{N-\ell+1}$ we admit the case $\xi=(1,0, \ldots, 0)$. This will correspond to row insertion into an initially empty word. With $e_{1}^{(k)}=(1,0, \ldots, 0)$ denoting the first unit $k$-vector, the notation and definition are now

$$
e_{1}^{(N-\ell+1)} \stackrel{b}{\downarrow} \quad \xi^{\prime} \quad \text { where } \quad \xi_{k}^{\prime}=\prod_{i=\ell}^{k} b_{i}, \quad \ell \leq k \leq N .
$$

This is consistent with (2.1) except that output $b^{\prime}$ is not defined and hence not displayed in the diagram above.

The next step is geometric row insertion of a word into a triangular array. For $N \geq 1$ let $\mathbb{T}_{N}=\left(z_{k \ell}\right.$ : $1 \leq \ell \leq k \leq N$, and $\left.z_{k \ell} \in(0, \infty)\right)$, i.e. the set of triangular arrays with positive real entries. The bottom picture of Figure 4 illustrates an element of $\mathbb{T}_{5}$. $\left(z_{k \ell}\right)$ consists of rows indexed by $k$ and southeast-pointing diagonals indexed by $\ell$.

Definition 2.2. Given $z \in \mathbb{T}_{N}$ and a word $b \in(0, \infty)^{N}$. Geometric row insertion of $b$ into $z$ outputs a new triangular array $z^{\prime} \in \mathbb{T}_{N}$. This procedure is denoted by

$$
z^{\prime}=z \leftarrow b
$$

and it consists of $N$ iterations of the basic row insertion. For $1 \leq \ell \leq N$ form words $z_{\ell}=\left(z_{\ell \ell}, \ldots, z_{N \ell}\right)$. Begin by setting $a_{1}=b$. Then for $\ell=1, \ldots, N$ recursively apply the map

$$
z_{\ell} \stackrel{a_{\ell}}{\stackrel{\downarrow}{\longrightarrow}} z_{\ell}^{\prime}
$$

from Definition 2.1, where $a_{\ell+1}=a_{\ell}^{\prime}$. The last output $a_{N+1}$ is empty. The new array $z^{\prime}=\left(z_{k \ell}^{\prime}: 1 \leq \ell \leq\right.$ $k \leq N)$ is formed from the words $z_{\ell}^{\prime}=\left(z_{\ell \ell}^{\prime}, \ldots, z_{N \ell}^{\prime}\right)$. Along the way the procedure constructs an auxiliary triangular array $a=\left(a_{k \ell}: 1 \leq \ell \leq k \leq N\right)$ with diagonals $a_{\ell}=\left(a_{\ell \ell}, \ldots, a_{N \ell}\right)$.

Definition 2.2 of $z^{\prime}=z \leftarrow b$ can be summarized by these equations:

$$
\begin{aligned}
a_{k, 1} & =b_{k} & \text { for } 1 \leq k \leq N \\
a_{k+1, \ell+1} & =a_{k+1, \ell} \frac{z_{k+1, \ell} z_{k, \ell}^{\prime}}{z_{k+1, \ell}^{\prime} z_{k, \ell}} & \text { for } 1 \leq \ell \leq k<N \\
z_{k, \ell}^{\prime} & =a_{k, \ell}\left(z_{k, \ell}+z_{k-1, \ell}^{\prime}\right) & \text { for } 1 \leq \ell<k \leq N \\
z_{k, k}^{\prime} & =a_{k, k} z_{k, k} & \text { for } 1 \leq k \leq N .
\end{aligned}
$$

This procedure is illustrated in Figure 1 when $N=3$.

Iteration of the insertion procedure defines a temporal evolution $z(n), n=0,1,2, \ldots$, of an array $z(n) \in$ $\mathbb{T}_{N}$. This evolution is driven by a semi-infinite matrix $d=\left(d_{n j}: n \geq 1,1 \leq j \leq N\right)$ of positive weights $d_{n j}$. We write $d^{[1, n]}=\left(d_{i j}: 1 \leq i \leq n, 1 \leq j \leq N\right)$ for the matrix of the first $n$ rows of $d$, and $d^{[n]}=\left(d_{n 1}, \ldots, d_{n N}\right)$ 


\begin{tabular}{|c|c|c|c|c|c|c|}
\hline & $a_{1}(1)$ & & $a_{1}(2)$ & & $a_{1}(3)$ & \\
\hline \multirow[t]{2}{*}{$z_{1}(0)$} & $\downarrow$ & $z_{1}(1)$ & $\downarrow$ & $z_{1}(2)$ & $\downarrow$ & $z_{1}(3)$ \\
\hline & $a_{2}(1)$ & & $a_{2}(2)$ & & $a_{2}(3)$ & \\
\hline \multirow[t]{2}{*}{$z_{2}(0)$} & $\downarrow$ & $z_{2}(1)$ & $\downarrow$ & $z_{2}(2)$ & $\downarrow$ & $z_{2}(3)$ \\
\hline & $a_{3}(1)$ & & $a_{3}(2)$ & & $a_{3}(3)$ & \\
\hline \multirow[t]{4}{*}{$z_{3}(0)$} & $\downarrow$ & $z_{3}(1)$ & $\downarrow$ & $z_{3}(2)$ & $\downarrow$ & $z_{3}(3)$ \\
\hline & $a_{4}(1)$ & & $a_{4}(2)$ & & $a_{4}(3)$ & \\
\hline & $\vdots$ & $\vdots$ & $\vdots$ & $\vdots$ & $\vdots$ & $\vdots$ \\
\hline & $a_{N}(1)$ & & $a_{N}(2)$ & & $a_{N}(3)$ & \\
\hline \multirow[t]{2}{*}{$z_{N}(0)$} & $\downarrow$ & $z_{N}(1)$ & $\downarrow$ & $z_{N}(2)$ & $\downarrow$ & $z_{N}(3)$ \\
\hline & $\emptyset$ & & $\emptyset$ & & $\emptyset$ & \\
\hline
\end{tabular}

Figure 2. Evolution of the array $z(n)$ in state space $\mathbb{T}_{N}$ over time $n=0,1,2, \ldots$ The initial state $z(0)$ is on the left edge. The inputs come from the $d$-array: $a_{1}(n)=d^{[n]}=$ $\left(d_{n, 1}, \ldots, d_{n, N}\right)$.

for the $n^{\text {th }}$ row of $d$. The temporal evolution is then defined by successive insertions of rows of $d$ into the initial array: given $z(0) \in \mathbb{T}_{N}$ and $d$, then iteratively for $n \geq 1$,

$$
z(n)=\left[z(n-1) \leftarrow d^{[n]}\right]=\left[z(0) \leftarrow d^{[1]} \leftarrow d^{[2]} \leftarrow \cdots \leftarrow d^{[n]}\right]
$$

Figure 2 illustrates.

Example 2.3. Let us illustrate the construction with a step-by-step example. (We use rational numbers, though the procedures apply more generally for non-negative reals.) First consider geometric row insertion of $b=(1,5)$ into $\xi=(3,2)$. By the procedure of $(2.1)$ we first compute the output $\xi_{1}^{\prime}=b_{1} \xi_{1}=1 \cdot 3=3$ and then $\xi_{2}^{\prime}=b_{2}\left(\xi_{1}^{\prime}+\xi_{2}\right)=5(3+2)=25$. Then we compute $b_{2}^{\prime}=b_{2} \frac{\xi_{2} \xi_{1}^{\prime}}{\xi_{1} \xi_{2}^{\prime}}=5 \frac{2 \cdot 3}{3 \cdot 25}=\frac{2}{5}$. This calculation is represented by the diagram

$$
(3,2) \underset{\left(\frac{2}{5}\right)}{\stackrel{(1,5)}{\downarrow}}(3,25) .
$$

We now illustrate geometric insertion of $a_{1}=(2,2,4)$ into the triangular array $z$ with $z_{1}=(4,1,3)$, $z_{2}=(3,7)$ and $z_{3}=(2)$. That is to say, that $z$ is given by

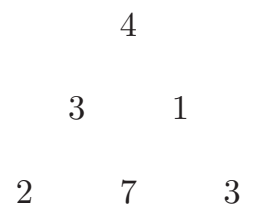

This insertion is performed by completing the following diagram.

$(2,2,4)$

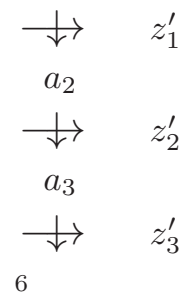




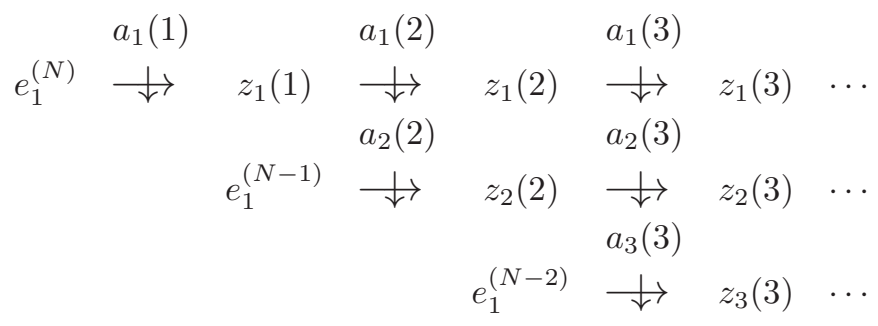

Figure 3. Evolution of the array $z(n)$ started from the empty array $z(0)=\emptyset . e_{1}^{(k)}$ represents the word $(1,0, \ldots, 0)$ of length $k$. By Proposition $2.5 z(n)$ is equal to the image $P_{n, N}\left(d^{[1, n]}\right)$ of the weight matrix $d^{[1, n]}=\left\{a_{1}(i)\right\}_{i=1}^{n}$ under the geometric RSK correspondence.

After calculating each insertion, starting from the top and sequentially going down, we arrive at the following diagram.

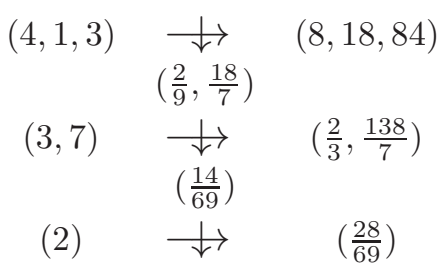

From the right-hand side of the diagram we read off the new array

8

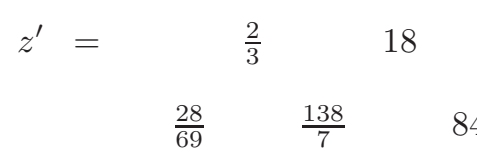

thus completing the insertion procedure.

Finally we consider the insertion process with an empty initial array. $N$ is still the fixed size parameter of the array. Initially $z(0)$ is empty which we denote by $z(0)=\emptyset$. The array grows by adding one new diagonal $z_{\ell}$ at each time. At time $n \in\{1, \ldots, N\}$, the already existing diagonals $z_{1}, \ldots, z_{n-1}$ are updated by inserting $a_{1}(n)$ and iterating as in (2.4), and a new diagonal $z_{n}$ is filled by inserting $a_{n}(n)$ according to (2.2). Consequently, at time $1 \leq n<N$, the currently defined array with strictly positive entries is $z(n)=\left\{z_{k \ell}(n): 1 \leq k \leq N, 1 \leq \ell \leq k \wedge n\right\}$. We consider the entries $\left\{z_{k \ell}(n): n<\ell \leq k \leq N\right\}$ undefined. At time $n=N$ the array is full, and after time $N$ its evolution continues according to (2.6). The evolution of $z(n)$ from $z(0)=\emptyset$ is illustrated by Figure 3 .

Remark 2.4. Instead of having truncated arrays in the evolution $\{z(n): 0 \leq n<N\}$ from $z(0)=\emptyset$, we could also choose to fill the undefined portion of the array with certain conventions that are consistent with the update rules. This would include use of 'singular values' 0 and $\infty$. For example, at time $0 \leq n<N$, diagonal $z_{n+1}(n)$ would equal $(1,0, \ldots, 0)$, in accordance with $(2.2)$. State space $\mathbb{T}_{N}$ would be replaced with a larger space $\mathbb{T}_{N}^{*}$ that contains $\emptyset$ and these other partially singular arrays. In this paper we will not use these conventions.

2.2. Geometric RSK via non-intersecting lattice paths. We turn to an alternative definition of the evolution in Figure 3 in terms of configurations of non-intersecting lattice paths. As before $N \geq 1$ is fixed and the input of the process is the semi-infinite matrix $d=\left(d_{i j}: i \geq 1,1 \leq j \leq N\right)$ of positive real weights. For each $n \geq 1$ form the $n \times N$ matrix $d^{[1, n]}=\left(d_{i j}: 1 \leq i \leq n, 1 \leq j \leq N\right)$. For $1 \leq \ell \leq k \leq N$ let $\Pi_{n, k}^{\ell}$ denote the set of $\ell$-tuples $\pi=\left(\pi_{1}, \ldots, \pi_{\ell}\right)$ of non-intersecting lattice paths in $\mathbb{Z}^{2}$ such that, for $1 \leq r \leq \ell, \pi_{r}$ is a lattice path from $(1, r)$ to $(n, k+r-\ell)$. A 'lattice path' only takes unit steps in the coordinate directions 
between nearest-neighbor lattice points of $\mathbb{Z}^{2}$ (i.e., up or right); non-intersecting means that paths do not touch. The weight of an $\ell$-tuple $\pi=\left(\pi_{1}, \ldots, \pi_{\ell}\right)$ of such paths is

$$
w t(\pi)=\prod_{r=1}^{\ell} \prod_{(i, j) \in \pi_{r}} d_{i j} .
$$

For $1 \leq \ell \leq k \leq N$ let

$$
\tau_{k, \ell}(n)=\sum_{\pi \in \Pi_{n, k}^{\ell}} w t(\pi)
$$

For $0 \leq n<\ell<k \leq N$ the set of paths $\Pi_{n, k}^{\ell}$ is empty and we take the empty sum to equal zero. At $\ell=k$ there is a unique $\ell$-tuple, and in fact we have the equation

$$
\tau_{k, \ell}(n)=\delta_{k, \ell} \tau_{k, n}(n) \quad \text { for } \quad 0 \leq n<\ell \leq k \leq N
$$

where $\delta_{k, \ell}$ is the Kronecker delta. For $\ell=0$ the right convention turns out to be $\tau_{k, 0}(n)=1$ for $1 \leq k \leq N$.

The array $z(n)=\left\{z_{k, \ell}(n): 1 \leq k \leq N, 1 \leq \ell \leq k \wedge n\right\}$ is now defined by

$$
z_{k, 1}(n) \cdots z_{k, \ell}(n)=\tau_{k, \ell}(n)
$$

The elements $\left(z_{k \ell}(n): n<\ell \leq k \leq N\right)$ we regard as undefined, even though strictly speaking one more element, namely $z_{n+1, n+1}(n)$, could be consistently defined as 1 . In the spirit of Remark 2.4 we could also replace the undefined array elements with particular singular values. See Figure 4 for an illustration.

We express the mapping (2.9) that defines $z(n)$ from $d^{[1, n]}$ as

$$
z(n)=P_{n, N}\left(d^{[1, n]}\right) .
$$

We come to the important point from Section 2.2 of [43] that row insertion into an empty array and this path construction define the same array $z(n)$. We postpone the proof of this proposition to the end of the section.

Proposition 2.5. Let $n, N \geq 1$. Set $z(n)=P_{n, N}\left(d^{[1, n]}\right)$ and

$$
\tilde{z}(n)=\emptyset \leftarrow d^{[1]} \leftarrow d^{[2]} \leftarrow \cdots \leftarrow d^{[n]} .
$$

Then $z(n)=\tilde{z}(n)$.

Let us discuss similarities with the classical RSK correspondence. Array $z=P_{n, N}\left(d^{[1, n]}\right)$ is the analogue of the $P$-tableau in the usual RSK correspondence. The analogue of the shape of this tableau is the bottom vector $\left(z_{N, 1}, \ldots, z_{N, N \wedge n}\right)$. The analogue of the $Q$-tableau is the array $w=Q_{n, N}\left(d^{[1, n]}\right)=P_{N, n}\left(\left(d^{[1, n]}\right)^{T}\right)$, where superscript $T$ denotes transpose. It is not difficult to see that the pair $(z, w)$ have the same 'shape', that is, $\left(z_{N, 1}, \ldots, z_{N, N \wedge n}\right)=\left(w_{n, 1}, \ldots, w_{n, N \wedge n}\right)$. Given this constraint, the pair $(z, w)$ can be identified with an $n \times N$ matrix $\tilde{d}$ defined by

$$
\tilde{d}_{i j}= \begin{cases}z_{i+j-1, i}, & 1 \leq i \leq n \wedge N, 1 \leq j \leq N-i+1 \\ w_{N+n-i-j+1, N-j+1}, & 1 \leq j \leq N, N-j+1 \leq i \leq n\end{cases}
$$

With this identification, the mapping $d^{[1, n]} \mapsto(z, w)$ is a bijection from the set of $n \times N$ matrices with strictly positive real entries onto itself, which one can refer to as the geometric RSK correspondence.

Proposition 2.5 shows that, as with the usual RSK correspondence, the ' $P$-tableau' $z(n)=P_{n, N}\left(d^{[1, n]}\right)$ can be defined recursively by inserting the rows of the matrix $d$ one after another. Then $w=Q_{n, N}(d)$ plays the role of a 'recording' tableau. With $z(k)=P_{k, N}\left(d^{[1, k]}\right)$ for $1 \leq k \leq n, w=Q_{n, N}(d)$ is given by $w_{k, \cdot}=z_{N, .}(k)$ for $1 \leq k \leq N$ : this is immediate from the definition.

Let us introduce some further conventions for the sequel. As before $z_{\ell}=\left(z_{\ell, \ell}, \ldots, z_{N, \ell}\right)$ is the $\ell^{\text {th }}$ diagonal of $z$, counting from right to left. The $k^{\text {th }}$ row of the array is denoted by $z^{[k]}=\left(z_{k 1}, \ldots, z_{k k}\right)$, and an array restricted to a range of rows is denoted by $z^{[a, b]}=\left(z_{k, \ell}: a \leq k \leq b, 1 \leq \ell \leq k\right)$. To discuss evolution of the array row by row it is convenient to have notation for spaces of rows. For $1 \leq k \leq N$ the $k^{\text {th }}$ row of an array in $\mathbb{T}_{N}$ lies in the space $\mathbb{Y}_{k}=\left(y_{\ell}: 1 \leq \ell \leq k\right.$, and $\left.y_{\ell} \in(0, \infty)\right)$, i.e., the space of vectors of length $k$ with positive real coordinates.

As the last item of this section we sketch the proof of Proposition 2.5 from [43]. 


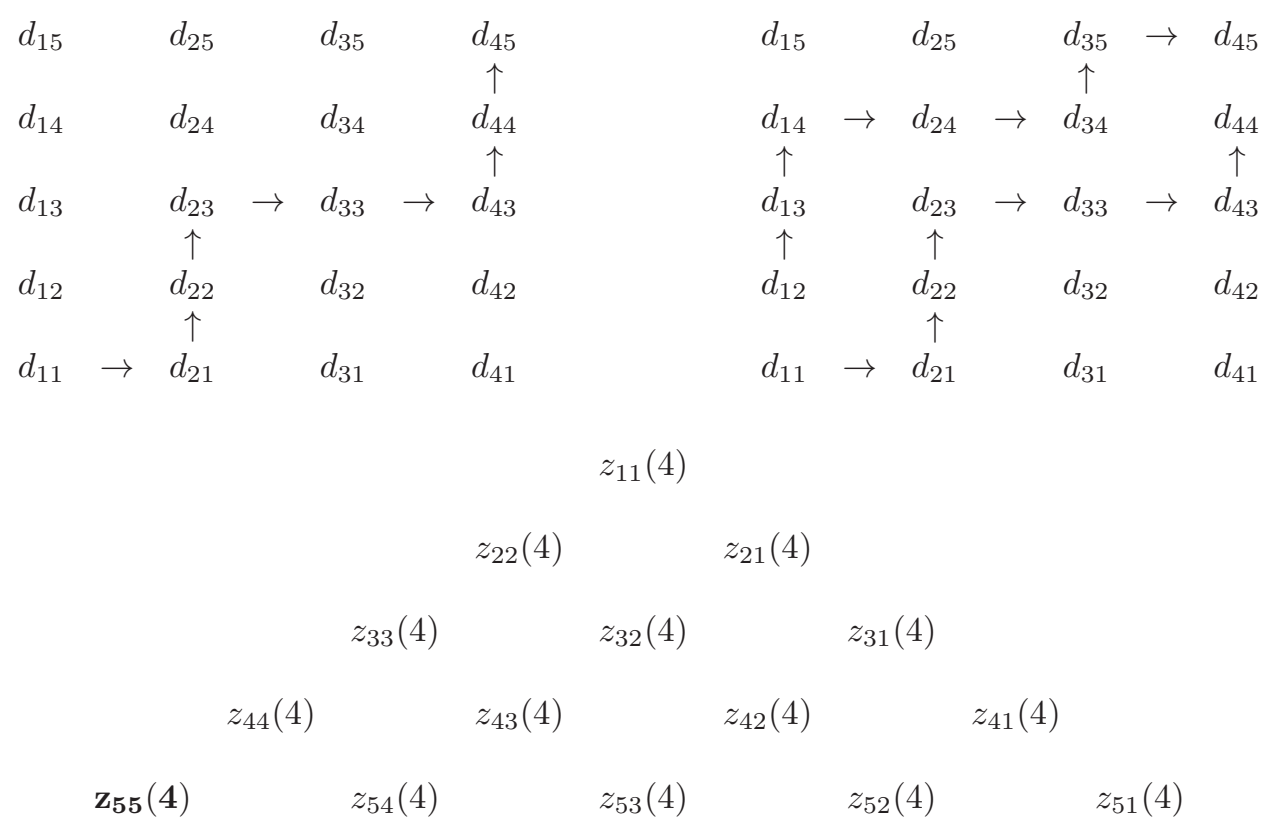

Figure 4. Illustration of the path construction for $n=4$ and $N=5$. Note that the matrix $d$ is presented in Cartesian coordinates. On the top left is an element $\pi$ of $\Pi_{n, N}^{1}$, that is an up-right path $\pi$ from $(1,1)$ to $(n, N)=(4,5)$. The weight of this path is $d_{11} d_{21} d_{22} d_{23} d_{33} d_{43} d_{44} d_{45}$. On the top right is an element $\pi=\left(\pi_{1}, \pi_{2}\right)$ of $\Pi_{n, N}^{2}$, a pair of nonintersecting up-right paths: $\pi_{1}$ from $(1,1)$ to $(n, N-1)=(4,4)$ and $\pi_{2}$ from $(1,2)$ to $(n, N)=(4,5)$. The weight of $\pi$ is $\left(d_{11} d_{21} d_{22} d_{23} d_{33} d_{43} d_{44}\right) \cdot\left(d_{12} d_{13} d_{14} d_{24} d_{34} d_{35} d_{45}\right)$. At the bottom is the array $z(n)=\left\{z_{k, \ell}(n)\right\}_{1 \leq \ell \leq k \leq N}$, at time $n=4$. We write this as $z(n)=P_{n, N}(d)$. The entry $z_{N, 1}(n)=z_{5,1}(4)$ is equal to the sum of the weights of all paths in $\Pi_{n, N}^{1}$. The product $z_{N, 1}(n) z_{N, 2}(n)=z_{5,1}(4) z_{5,2}(4)$ is equal to the sum of the weights of all elements in $\Pi_{n, N}^{2}$. The rest of the array is determined similarly via (2.9). We regard the boldface element $\mathbf{z}_{\mathbf{5 5}}(\mathbf{4})$ as not yet defined at time $n=4$ as explained after (2.9).

Proof of Proposition 2.5. The connection between $z(n)$ and $\tilde{z}(n)$ goes via the variables $\tau_{k \ell}(n)$ and a matrix formalism developed in [43].

For an $N$-vector $x=\left(x_{1}, \ldots, x_{N}\right)$ define an upper triangular $N \times N$ matrix

$$
H(x)=\sum_{1 \leq i \leq j \leq N} x_{i} x_{i+1} \cdots x_{j} E_{i, j}
$$

where $E_{i, j}$ is the $N \times N$ matrix with a unique 1 in the $(i, j)$-position and zeroes elsewhere. For a fixed $n$, define the product

$$
H=H\left(d^{[1]}\right) H\left(d^{[2]}\right) \cdots H\left(d^{[n]}\right) .
$$

A key fact [43, Prop. 1.3] is that the $\tau_{k \ell}(n)$ 's give certain minor determinants of $H: \tau_{k \ell}(n)=\operatorname{det} H_{[k-\ell+1, k]}^{[1, \ell]}$ where the superscript specifies the range of rows and the subscript the range of columns in the minor.

On the other hand, the row insertion procedure can be encoded with $H$-type matrices. Let $1 \leq m \leq N$ and introduce this further definition for an $(N-m+1)$-vector $x=\left(x_{m}, x_{m+1}, \ldots, x_{N}\right)$ :

$$
H_{m}(x)=\sum_{1 \leq i<m} E_{i, i}+\sum_{m \leq i \leq j \leq N} x_{i} x_{i+1} \cdots x_{j} E_{i, j} .
$$

In particular, $H_{1}(x)=H(x)$. 
Set $M=n \wedge N$. With $H$ as in (2.12) above, consider the equation

$$
H=H_{M}\left(\eta_{M}\right) \cdots H_{2}\left(\eta_{2}\right) H_{1}\left(\eta_{1}\right)
$$

for unknown vectors $\eta_{\ell}=\left(\eta_{k, \ell}\right)_{\ell \leq k \leq N}, \ell=1,2, \ldots, M$. This equation is uniquely solved by [43, Thm. 2.4]

$$
\eta_{\ell, \ell}=\frac{\tau_{\ell, \ell}(n)}{\tau_{\ell, \ell-1}(n)}, \quad \eta_{k, \ell}=\frac{\tau_{k, \ell}(n) \tau_{k-1, \ell-1}(n)}{\tau_{k, \ell-1}(n) \tau_{k-1, \ell}(n)} \quad \text { for } \ell<k \leq N .
$$

Equation (2.14) encodes the row insertion procedure but in different variables [43, eqn. (2.38)-(2.40)]. Namely, the $\eta$-variables are the ratios of the $\tilde{z}$-variables defined by (2.11):

$$
\text { for } 1 \leq \ell \leq M: \quad \eta_{\ell, \ell}=\tilde{z}_{\ell, \ell}(n), \quad \eta_{k, \ell}=\frac{\tilde{z}_{k, \ell}(n)}{\tilde{z}_{k-1, \ell}(n)} \quad \text { for } \ell<k \leq N
$$

Combining (2.15)-(2.16) for $\tilde{z}$ with (2.9) for $z$ gives

$$
\tilde{z}_{k, \ell}(n)=\eta_{\ell, \ell} \cdots \eta_{k, \ell}=\frac{\tau_{k, \ell}(n)}{\tau_{k, \ell-1}(n)}=z_{k, \ell}(n) \quad \text { for } 1 \leq \ell \leq M, \ell \leq k \leq N
$$

\section{Geometric RSK with RAndom input}

Given an initial (possibly random) state $z(0) \in \mathbb{T}_{N}$ and a weight matrix $d$ composed of independent random rows $d^{[i]}$, with $z(0)$ and $d$ independent, Proposition 2.5 shows that $z(n)=P_{n, N}\left(d^{[1, n]}\right)$ has the structure of a Markov process with time parameter $n$. The exact form of the transition kernel depends on the distribution of the rows $d^{[i]}$ and can be explicitly written down by appealing to the recursion of Definition 2.1. We do this for a particular solvable distribution on the elements $d_{i j}$ that we now introduce.

Definition 3.1. Let $\theta$ be a positive real. A random variable $X$ has inverse-gamma distribution with parameter $\theta>0$ if it is supported on the positive reals where it has distribution

$$
\mathbb{P}(X \in d x)=\frac{1}{\Gamma(\theta)} x^{-\theta-1} \exp \left\{-\frac{1}{x}\right\} d x .
$$

We abbreviate this $X \sim \Gamma^{-1}(\theta)$.

Definition 3.2. An inverse-gamma weight matrix, with respect to a parameter matrix $\gamma=\left(\gamma_{i, j}>0: i \geq\right.$ $1,1 \leq j \leq N)$, is a matrix of positive weights $\left(d_{i, j}: i \geq 1,1 \leq j \leq N\right)$ such that the entries are independent random variables and $d_{i, j} \sim \Gamma^{-1}\left(\gamma_{i, j}\right)$. We call a parameter matrix $\gamma$ solvable if $\gamma_{i, j}=\hat{\theta}_{i}+\theta_{j}>0$ for real parameters $\left(\hat{\theta}_{i}: i \geq 1\right)$ and $\left(\theta_{j}: 1 \leq j \leq N\right)$. In this case we also refer to the associated weight matrix as solvable. Column $n$ of the parameter matrix $\gamma$ is denoted by $\gamma^{[n]}=\left(\gamma_{n, j}\right)_{1 \leq j \leq N}$. We denote the vector $\theta=\left(\theta_{j}: 1 \leq j \leq N\right)$ for later use.

The intertwining properties we discuss in Section 3.1 are the reason we restrict to inverse-gamma distributed weights and the reason for the particular form $\gamma_{i, j}=\hat{\theta}_{i}+\theta_{j}$.

The transition kernel for the Markov chain $z(n)$ on the state space $\mathbb{T}_{N}$ resulting from applying the geometric RSK correspondence to a solvable inverse-gamma weight matrix is denoted by $\Pi_{\gamma^{[n]}}^{N}(z, d \tilde{z})$. This represents the time $n$ transition $z(n-1) \rightarrow z(n)$.

To explicitly state this kernel, it turns out useful to exploit another structural property of the image of the geometric RSK with independent weights $d_{n, k}$ : the rows of the array $z(n)$ form a Markov chain (indexed from top to bottom) with respect to adding columns to the weight matrix $d$. For this purpose let us denote $z=z(n-1)$ and $\tilde{z}=z(n)$. We begin at the top of the array. The singleton top row (denoted by $y$ ) of $z$ is updated at time $n$ by the transition kernel

$$
P_{\gamma^{[n]}}^{1}(y, d \tilde{y})=\Gamma\left(\gamma_{n, 1}\right)^{-1}\left(\frac{y_{1}}{\tilde{y}_{1}}\right)^{\gamma_{n, 1}} \exp \left\{-\frac{y_{1}}{\tilde{y}_{1}}\right\} \frac{d \tilde{y}_{1}}{\tilde{y}_{1}} .
$$

This simply encodes $\tilde{y}=d_{n, 1} y$ with $d_{n, 1} \sim \Gamma^{-1}\left(\gamma_{n, 1}\right)$.

Now we move down along the rows of the array (recall $z^{[k-1]}=\left(z_{k-1, \ell}\right)_{1 \leq \ell \leq k-1}$ and likewise for $\tilde{z}^{[k-1]}$ ). Given both the initial and updated row $k-1,\left(z^{[k-1]}, \tilde{z}^{[k-1]}\right)$, from $(2.5)$ we read off the time $n$ rule for 
updating row $k$ from $z^{[k]}=\left(z_{k, \ell}\right)_{1 \leq \ell \leq k}$ to $\tilde{z}^{[k]}=\left(\tilde{z}_{k, \ell}\right)_{1 \leq \ell \leq k}$. The new input weight is $a_{k, 1}=d_{n, k} \sim$ $\Gamma^{-1}\left(\gamma_{n, k}\right)$, and the equations are

$$
\begin{aligned}
& \tilde{z}_{k, 1}=a_{k, 1}\left(z_{k, 1}+\tilde{z}_{k-1,1}\right) \\
& \tilde{z}_{k, \ell}=\frac{z_{k, \ell-1} \tilde{z}_{k-1, \ell-1}}{z_{k-1, \ell-1}} \cdot \frac{z_{k, \ell}+\tilde{z}_{k-1, \ell}}{z_{k, \ell-1}+\tilde{z}_{k-1, \ell-1}} \quad \text { for } 2 \leq \ell \leq k-1, \\
& \tilde{z}_{k, k}=\frac{z_{k, k} z_{k, k-1} \tilde{z}_{k-1, k-1}}{\left(z_{k, k-1}+\tilde{z}_{k-1, k-1}\right) z_{k-1, k-1}} .
\end{aligned}
$$

Equations (3.3) above show that $\tilde{z}^{[k]}$ is an explicit function of $z^{[k-1]}, z^{[k]}, \tilde{z}^{[k-1]}$ and the (random) weight $a_{k, 1}$. Taking the push-forward of the measure on $a_{k, 1}$ under this function yields a probability distribution on $\tilde{z}^{[k]}$, as a function of $\left(z^{[k-1]}, z^{[k]}, \tilde{z}^{[k-1]}\right)$. We denote this probability distribution by the stochastic kernel $L_{\gamma_{n, k}}^{k}\left(\left(z^{[k-1]}, z^{[k]} ; \tilde{z}^{[k-1]}\right), d \tilde{z}^{[k]}\right)$ from $\mathbb{Y}_{k-1} \times \mathbb{Y}_{k} \times \mathbb{Y}_{k-1}$ into $\mathbb{Y}_{k}$. Explicitly it is given as follows in terms of its integral against a bounded Borel test function $g$ on $\mathbb{Y}_{k}$ :

$$
\begin{aligned}
\int_{(0, \infty)^{k}} g(\tilde{y}) L_{\gamma_{n, k}}^{k}((x, y ; \tilde{x}), d \tilde{y})=\int_{(0, \infty)} \frac{d \tilde{y}_{1}}{\tilde{y}_{1}} \Gamma\left(\gamma_{n, k}\right)^{-1}\left(\frac{y_{1}+\tilde{x}_{1}}{\tilde{y}_{1}}\right)^{\gamma_{n, k}} \exp \left\{-\frac{y_{1}+\tilde{x}_{1}}{\tilde{y}_{1}}\right\} \\
\times g\left(\tilde{y}_{1},\left\{\frac{y_{\ell-1} \tilde{x}_{\ell-1}}{x_{\ell-1}} \cdot \frac{y_{\ell}+\tilde{x}_{\ell}}{y_{\ell-1}+\tilde{x}_{\ell-1}}\right\}_{2 \leq \ell \leq k-1}, \frac{y_{k} y_{k-1} \tilde{x}_{k-1}}{x_{k-1}\left(y_{k-1}+\tilde{x}_{k-1}\right)}\right) .
\end{aligned}
$$

Now we can write down the kernel for the evolution of the array. The kernel $\Pi_{\gamma^{[n]}}^{N}(z, d \tilde{z})$ for the transition from $z=z(n-1)$ to $\tilde{z}=z(n)$ on the space $\mathbb{T}_{N}$ is defined inductively on $N$. For $N=1$ set $\Pi_{\gamma^{[n]}}^{1}=P_{\gamma^{[n]}}^{1}$, and for $N \geq 2$

$$
\Pi_{\gamma^{[n]}}^{N}\left(z^{[1, N]}, d \tilde{z}^{[1, N]}\right)=\Pi_{\gamma^{[n]}}^{N-1}\left(z^{[1, N-1]}, d \tilde{z}^{[1, N-1]}\right) L_{\gamma_{n, N}}^{N}\left(\left(z^{[N-1]}, z^{[N]} ; \tilde{z}^{[N-1]}\right), d \tilde{z}^{[N]}\right) .
$$

3.1. Intertwining relation. Let us first recall a well-known criterion for a function of a Markov chain to retain the Markov property (see, for example, [54]). Consider a measurable transformation $\phi: T \rightarrow S$ where $(T, \mathcal{T})$ and $(S, \mathcal{S})$ are measurable spaces. Given Markov transition kernels $\Pi_{n}$ on $T$ one forms a Markov process $z(n)$ which has a given initial distribution $z(0)$ and transitions between $z(n-1)$ to $z(n)$ via $\Pi_{n}$. The process $\{z(n)\}_{n \geq 0}$ is Markovian with respect to its own filtration. The question is, under what conditions is $y(n)=\phi(z(n))$ Markovian under its own filtration $\sigma\{y(0), \ldots, y(n)\}$, and what is the associated transition kernel $\bar{P}_{n}: S \rightarrow S$ ?

In order to answer this question we introduce an intertwining kernel $\bar{K}: S \rightarrow T$. (The reason $\bar{P}$ and $\bar{K}$ have bars is that one often initially deals with unnormalized kernels and then normalizes them to be probability measures in their second variable.)

Proposition 3.3. Assume that there exist $\bar{P}_{n}$ and $\bar{K}$ (which does not depend on $n$ ) satisfying

(1) for all $y \in S, \bar{K}\left(y, \phi^{-1}(y)\right)=1$,

(2) for all $n, \bar{K} \Pi_{n}=\bar{P}_{n} \bar{K}$.

Then, for any initial (possibly random) state $y^{0} \in S$, if one initializes the Markov chain $z(n)$ with $z(0)$ distributed according to the measure $\bar{K}\left(y^{0}, \cdot\right)$, one has these properties:

(i) For all $y \in S$ and all bounded Borel functions $f$ on $T$,

$$
\mathbb{E}[f(z(n)) \mid y(0), \ldots, y(n-1), y(n)=y]=(\bar{K} f)(y) .
$$

(ii) The process $y(n)=\phi(z(n))$ is Markov in its own filtration $\sigma\{y(0), \ldots, y(n)\}$ with transition kernel $\bar{P}_{n}$.

Return to geometric RSK with the solvable inverse-gamma weight matrix. Take $T$ above to be $\mathbb{T}_{N}$ and $S$ to be $\mathbb{Y}_{N}$. Let $\phi: \mathbb{T}_{N} \rightarrow \mathbb{Y}_{N}$ project $z \in \mathbb{T}_{N}$ onto its bottom row. It is easier to first introduce unnormalized kernels and prove intertwining, and then to normalize them to apply the above results. 
Define a time $n$ positive kernel on $\mathbb{Y}_{N}$ by

$$
P_{\gamma^{[n]}}^{N}(y, d \tilde{y})=\prod_{i=1}^{N-1} \exp \left\{-\frac{\tilde{y}_{i+1}}{y_{i}}\right\} \prod_{j=1}^{N}\left(\Gamma\left(\gamma_{n, j}\right)^{-1}\left(\frac{y_{j}}{\tilde{y}_{j}}\right)^{\gamma_{n, j}} \exp \left\{-\frac{y_{j}}{\tilde{y}_{j}}\right\} \frac{d \tilde{y}_{j}}{\tilde{y}_{j}}\right) .
$$

Define a positive intertwining kernel from $\mathbb{Y}_{N}$ to $\mathbb{T}_{N}$ by

$$
K_{\theta}^{N}(y, d z)=\prod_{1 \leq \ell \leq k<N}\left(\frac{z_{k, \ell}}{z_{k+1, \ell}}\right)^{\theta_{k+1}-\theta_{\ell}} \exp \left(-\frac{z_{k, \ell}}{z_{k+1, \ell}}-\frac{z_{k+1, \ell+1}}{z_{k, \ell}}\right) \frac{d z_{k, \ell}}{z_{k, \ell}} \prod_{\ell=1}^{N} \delta_{y_{\ell}}\left(d z_{N \ell}\right)
$$

where $\delta_{y}\left(d z_{i j}\right)$ is the Dirac delta measure at $y . K_{\theta_{1}}^{1}$ is the identity kernel. Observe that $K_{\theta}^{N}$ only depends on the column parameters $\theta$ which do not change with the time index $n$. Definition 3.2 stipulated the form $\gamma_{i, j}=\hat{\theta}_{i}+\theta_{j}$ for the solvable parameter matrix in order to make the intertwining work. The intertwining itself does also work with a time-dependent $K$-kernel. But in our case computations reveal that application of Proposition 3.3 requires a time-independent $K$ and we are not permitted any more general $\left(\gamma_{i, j}\right)$.

$P_{\gamma^{[n]}}^{1}$ is a stochastic kernel (i.e., normalized to have measure one) and represents the update $\tilde{z}_{1,1}=a_{1,1} z_{1,1}$. But for $N \geq 2, P_{\gamma}^{N[n]}$ is substochastic. This is evident because the second product is the stochastic kernel of independent inverse-gamma distributed multiplicative jumps, while the first product is a killing potential. A Doob $h$-transform (or ground-state transform) will suffice to renormalize this kernel as well as the intertwining kernel.

The main algebraic content of the integrability or solvability of geometric RSK is:

Proposition 3.4. The following intertwining relation holds at all times $n \geq 1$ :

$$
P_{\gamma}^{N[n]} K_{\theta}^{N}=K_{\theta}^{N} \Pi_{\gamma}^{N}{ }^{[n]},
$$

where both sides are operators from $\mathbb{Y}_{N}$ to $\mathbb{T}_{N}$.

This is proved in Section 5.1.

Remark 3.5. The above proposition along with the existence of the kernels $P_{\gamma[n]}^{N}$ and $K_{\theta}^{N}$ are the key to the main argument of this paper. A priori, the existence of such kernels is not guaranteed. The inspiration behind finding these kernels came from the related work [44] (in which the kernel $K_{\theta}^{N}$ also appears and plays a similarly important role) as well as earlier work in which analogous results were obtained in the context of the usual RSK correspondence $[45,46,10,11]$ (see also $\S \S 3.4$ below). In the context of the RSK correspondence with geometric or exponentially distributed weights, the kernel $K_{\theta}^{N}(y, d z)$ is replaced by the indicator function over Gelfand-Tsetlin patterns (interlacing triangular arrays) $z$ with bottom row $y$, with an exponential factor depending on the type of the pattern; and the kernel $P_{\gamma[n]}^{N}(y, d \tilde{y})$ is replaced by the substochastic kernel of independent geometrically or exponentially distributed additive jumps from $y$ to $\tilde{y}$ subject to the constraint that $\tilde{y}$ is interlaced with $y$.

The kernels above are not normalized. However, using the intertwining relation it is now simple to determine the necessary normalizing functions. For $y \in \mathbb{Y}_{N}$, define

$$
w_{\theta}^{N}(y)=\int_{\mathbb{T}_{N}} K_{\theta}^{N}(y, d z)
$$

Integrating the intertwining (3.8) yields the eigenfunction relation

$$
P_{\gamma^{[n]}}^{N} w_{\theta}^{N}=w_{\theta}^{N}
$$

Thus we can define a stochastic kernel on $\mathbb{Y}_{N}$ by

$$
\bar{P}_{\gamma^{[n]}}^{N}(y, d \tilde{y})=\frac{w_{\theta}^{N}(\tilde{y})}{w_{\theta}^{N}(y)} P_{\gamma}^{N}{ }^{[n]}(y, d \tilde{y})
$$

and from $\mathbb{Y}_{N}$ to $\mathbb{T}_{N}$ by

$$
\bar{K}_{\theta}^{N}(y, d z)=\frac{1}{w_{\theta}^{N}(y)} K_{\theta}^{N}(y, d z)
$$


The kernel $\bar{K}_{\theta}^{N}(y, d z)$ should be interpreted as the distribution of the pattern in $\mathbb{T}_{N}$ conditioned on the bottom row $z^{[N]}$ being equal to $y$. From the previous proposition follows:

Corollary 3.6. The following intertwining relation holds at all times $n \geq 1$ :

$$
\bar{P}_{\gamma^{[n]}}^{N} \bar{K}_{\theta}^{N}=\bar{K}_{\theta}^{N} \Pi_{\gamma^{[n]}}^{N} \text {, }
$$

where both sides are operators from $\mathbb{Y}_{N}$ to $\mathbb{T}_{N}$.

3.2. Whittaker functions. For the next stage, note that the kernels above remain perfectly well-defined if we allow parameter vector $\theta$ to be complex. The probabilistic meanings are lost but the intertwining continues to work. For $y \in(0, \infty)^{N}$ and $\lambda \in \mathbb{C}^{N}$, define

$$
M_{\lambda}^{N}(y, d z)=\prod_{i=1}^{N} y_{i}^{-\lambda_{i}} K_{\lambda}^{N}(y, d z)
$$

and

$$
\Psi_{\lambda}^{N}(y)=\int_{\mathbb{T}_{N}} M_{\lambda}^{N}(y, d z)=\prod_{i=1}^{N} y_{i}^{-\lambda_{i}} w_{\lambda}^{N}(y) .
$$

The functions $\Psi_{\lambda}^{N}$, well-defined for any $\lambda \in \mathbb{C}^{N}$, are class-one $G L(N, \mathbb{R})$-Whittaker functions (in multiplicative variables). They arise in various contexts: they are eigenfunctions of the quantum Toda lattice (when expressed in additive variables $x_{i}=\log y_{i}$ ) and can be represented as particular matrix elements of infinite-dimensional representations of $\mathfrak{g l}(N)$ [38]; they also arise in the harmonic analysis of automorphic forms on $G L(N, \mathbb{R})$ [16]. The integral representation (3.15) is due to Givental [30]. It is known [56, 35] that the integral transform

$$
\hat{f}(\lambda)=\int_{(0, \infty)^{N}} \prod_{i=1}^{N} \frac{d y_{i}}{y_{i}} f(y) \Psi_{\lambda}^{N}(y)
$$

defines an isometry of $L_{2}\left((0, \infty)^{N}, \prod_{i} d y_{i} / y_{i}\right)$ onto $L_{2}^{\text {sym }}\left(\iota \mathbb{R}^{N}, s_{N}(\lambda) d \lambda\right)$, where $L_{2}^{\text {sym }}$ is the space of $L^{2}$ functions which are symmetric in their variables, $\iota=\sqrt{-1}$ is the imaginary unit and

$$
s_{N}(\lambda)=\frac{1}{(2 \pi \iota)^{N} N !} \prod_{j \neq k} \Gamma\left(\lambda_{j}-\lambda_{k}\right)^{-1} .
$$

The inversion formula is

$$
\check{g}(y)=\int_{\iota \mathbb{R}^{N}} g(\lambda) \overline{\Psi_{\lambda}^{N}(y)} s_{N}(\lambda) d \lambda .
$$

In particular, the Plancherel formula

$$
\int_{(0, \infty)^{N}} f(y) \overline{g(y)} \prod_{i=1}^{N} \frac{d y_{i}}{y_{i}}=\int_{\iota \mathbb{R}^{N}} \hat{f}(\lambda) \overline{\hat{g}(\lambda)} s_{N}(\lambda) d \lambda
$$

holds for functions $f, g \in L^{2}\left((0, \infty)^{N}, \prod d y_{i} / y_{i}\right)$.

We also have the Whittaker integral identity [17, 61, 29], for $s>0$ and $\lambda, \nu \in \mathbb{C}^{N}$,

$$
\int_{(0, \infty)^{N}} e^{-s y_{1}} \Psi_{\lambda}^{N}(y) \Psi_{\nu}^{N}(y) \prod_{i=1}^{N} \frac{d y_{i}}{y_{i}}=s^{\sum\left(\lambda_{i}+\nu_{i}\right)} \prod_{i, j} \Gamma\left(-\lambda_{i}-\nu_{j}\right) .
$$

Using $\Psi_{\theta}(y)=\Psi_{-\theta}\left(y^{\prime}\right)$, where $y_{i}^{\prime}=y_{N-i+1}^{-1}$, this is equivalent to

$$
\int_{(0, \infty)^{N}} e^{-s y_{N}^{-1}} \Psi_{\lambda}^{N}(y) \Psi_{\nu}^{N}(y) \prod_{i=1}^{N} \frac{d y_{i}}{y_{i}}=s^{-\sum\left(\lambda_{i}+\nu_{i}\right)} \prod_{i, j} \Gamma\left(\lambda_{i}+\nu_{j}\right) .
$$

Note that if, for $z \in \mathbb{T}_{N}$, we define $x_{i}(z)$ by

$$
\prod_{i=1}^{k} x_{i}(z)=\prod_{i=1}^{k} z_{k, i}, \quad k=1, \ldots, N
$$


then

$$
M_{\theta}^{N}(y, d z)=\prod_{i=1}^{N} x_{i}(z)^{-\theta_{i}} M^{N}(y, d z)
$$

where

$$
M^{N}(y, d z)=\prod_{1 \leq \ell \leq k<N} \exp \left(-\frac{z_{k, \ell}}{z_{k+1, \ell}}-\frac{z_{k+1, \ell+1}}{z_{k, \ell}}\right) \frac{d z_{k, \ell}}{z_{k, \ell}} \prod_{\ell=1}^{N} \delta_{y_{\ell}}\left(d z_{N \ell}\right) .
$$

For $n \geq 0$ and $i=1, \ldots, N$ write $x_{i}(n)=x_{i}(z(n))$. Then $x_{i}(n)$ is a multiplicative random walk:

$$
x_{i}(n)=\left(\prod_{m=1}^{n} d_{m, i}\right) x_{i}(0) .
$$

3.3. Main theorems. We are prepared to state the two main theorems of the paper. They are proved in Sections 5.2 and 5.3, respectively. The first result is concerned with the solvability of the $\phi$ projection of the $z(n)$ Markov chain corresponding to the recursive system in equation (2.5).

Theorem 3.7. Fix a solvable inverse-gamma weight matrix defined in terms of parameters $\left(\hat{\theta}_{m}: m \geq 1\right)$ and $\left(\theta_{j}: 1 \leq j \leq N\right)$ and assume (without loss of generality) that $\theta_{j}<0<\hat{\theta}_{m}$ for all $j, m$. Let $y(0)$ be a random or deterministic initial state in $\mathbb{Y}_{N}$ and let the initial distribution of $z(0)$ be $\bar{K}_{\theta}^{N}(y(0), \cdot)$.

(i) The sequence of random variables $y(n)=\phi(z(n)), n \geq 0$, is a Markov chain with respect to its own filtration, with state space $\mathbb{Y}_{N}$, initial state $y(0)$ and time $n$ transition kernel $\bar{P}_{\gamma^{[n]}}^{N}$.

(ii) For a bounded Borel function $f$ on $\mathbb{T}_{N}$ and $y \in \mathbb{Y}_{N}$

$$
E[f(z(n)) \mid y(0), \ldots, y(n-1), y(n)=y]=\int_{\mathbb{T}_{N}} \bar{K}_{\theta}^{N}(y, d z) f(z) .
$$

(iii) For $\lambda \in \mathbb{C}^{N}$

$$
E\left[\prod_{i=1}^{N} x_{i}(n)^{-\lambda_{i}} \mid y(0), \ldots, y(n-1), y(n)=y\right]=\frac{\Psi_{\theta+\lambda}^{N}(y)}{\Psi_{\theta}^{N}(y)} .
$$

(iv) For an initial state $y^{0} \in \mathbb{Y}_{N}$ and time $\geq 1$, let $\mu_{n}^{N}\left(y^{0}, d y\right)$ denote the probability distribution of the time-n state $y(n)$. Then for all $\lambda \in \iota \mathbb{R}^{N}$,

$$
\int_{(0, \infty)^{N}} \frac{\Psi_{\lambda}^{N}(y)}{\Psi_{\theta}^{N}(y)} \mu_{n}^{N}\left(y^{0}, d y\right)=\frac{\Psi_{\lambda}^{N}\left(y^{0}\right)}{\Psi_{\theta}^{N}\left(y^{0}\right)} \prod_{m=1}^{n} \prod_{i=1}^{N} \frac{\Gamma\left(\hat{\theta}_{m}+\lambda_{i}\right)}{\Gamma\left(\theta_{i}+\hat{\theta}_{m}\right)} .
$$

Moreover, for any continuous, compactly supported function $f$ on $(0, \infty)^{N}$ we have

$$
\int_{\mathbb{R}_{+}^{N}} f(y) \mu_{n}^{N}\left(y^{0}, d y\right)=\int_{\iota \mathbb{R}^{N}} d \lambda s_{N}(\lambda) \frac{\Psi_{\lambda}^{N}\left(y^{0}\right)}{\Psi_{\theta}^{N}\left(y^{0}\right)}\left(\int_{\mathbb{R}_{+}^{N}} f(y) \Psi_{\theta}^{N}(y) \Psi_{-\lambda}^{N}(y) \prod_{i} \frac{d y_{i}}{y_{i}}\right) \prod_{m=1}^{n} \prod_{i=1}^{N} \frac{\Gamma\left(\hat{\theta}_{m}+\lambda_{i}\right)}{\Gamma\left(\theta_{i}+\hat{\theta}_{m}\right)} .
$$

Remark 3.8. Note that, given part (i) of the above theorem, (3.27) shows that one may diagonalize the transition kernel via the eigenfunction equation

$$
\bar{P}_{\gamma^{[n]}}^{N} \frac{\Psi_{\lambda}^{N}}{\Psi_{\theta}^{N}}=\left(\prod_{j=1}^{N} \frac{\Gamma\left(\hat{\theta}_{n}+\lambda_{j}\right)}{\Gamma\left(\theta_{j}+\hat{\theta}_{n}\right)}\right) \frac{\Psi_{\lambda}^{N}}{\Psi_{\theta}^{N}}
$$

which can also be seen directly from the intertwining relation (3.8), cf. (3.10). By applying the completeness relation resulting from the $L^{2}$ isometry, this identity characterizes the transition kernel.

Next we specialize the above result to the Markov chain $y(n)$ that comes from the evolving shape of the geometric RSK array $P_{n, N}\left(d^{[1, n]}\right)$ of (2.10). This is the case of the empty initial array. We can capture this situation by taking a somewhat delicate limit of the initial state $y^{0}$. This is our second main result. 
Theorem 3.9. Fix a solvable inverse-gamma weight matrix defined in terms of parameters $\left(\hat{\theta}_{m}: m \geq 1\right)$ and $\left(\theta_{j}: 1 \leq j \leq N\right)$ and assume (without loss of generality) that $\theta_{j}<0<\hat{\theta}_{m}$ for all $j, m$.

Consider the array $\rho=\left(\rho_{k, \ell}\right)_{1 \leq \ell \leq k \leq N}$ with

$$
\rho_{k}=\left(\rho_{k, \ell}\right)_{1 \leq \ell \leq k}=\left(\frac{k-1}{2}, \frac{k-1}{2}-1, \ldots,-\frac{k-1}{2}\right),
$$

for $1 \leq k \leq N$. Let $y^{0, M}=\left(e^{-M \rho_{N, \ell}}\right)_{1 \leq \ell \leq N}$ and $n \geq N$. Then

(i) $A s M \rightarrow \infty$, the probability distribution $\mu_{n}^{N}\left(y^{0, M}, d y\right)$ converges weakly to a distribution $\mu_{n}^{N}(d y)$ characterized by

$$
\int_{\mathbb{R}_{+}^{N}} f(y) \mu_{n}^{N}(d y)=\int_{\iota \mathbb{R}^{N}} d \lambda s_{N}(\lambda)\left(\int_{\mathbb{R}_{+}^{N}} f(y) \Psi_{\theta}(y) \Psi_{-\lambda}(y) \prod_{i} \frac{d y_{i}}{y_{i}}\right) \prod_{m=1}^{n} \prod_{i=1}^{N} \frac{\Gamma\left(\hat{\theta}_{m}+\lambda_{i}\right)}{\Gamma\left(\theta_{i}+\hat{\theta}_{m}\right)},
$$

for any continuous, compactly supported function $f$ on $(0, \infty)^{N}$.

(ii) The Laplace transform of the projection of $\mu_{n}^{N}(d y)$ on the first coordinate is given by

$$
\int_{(0, \infty)^{N}} e^{-s y_{1}} \mu_{n}^{N}(d y)=\int_{\iota \mathbb{R}^{N}} d \lambda s^{\sum_{i=1}^{N}\left(\theta_{i}-\lambda_{i}\right)} \prod_{1 \leq i, j \leq N} \Gamma\left(\lambda_{i}-\theta_{j}\right) \prod_{m=1}^{n} \prod_{i=1}^{N} \frac{\Gamma\left(\lambda_{i}+\hat{\theta}_{m}\right)}{\Gamma\left(\theta_{i}+\hat{\theta}_{m}\right)} s_{N}(\lambda),
$$

where the poles of the functions $\Gamma\left(\lambda_{i}-\theta_{j}\right)$ and $\Gamma\left(\lambda_{i}+\hat{\theta}_{m}\right)$ are not encountered due to the assumed condition that $\hat{\theta}_{m}>0$ for all $m$ and $\theta_{j}<0$ for all $j$.

(iii) The measure $\mu_{n}^{N}(d y)$ is the distribution of the bottom row $y(n)$, given that the process begins with the empty array. In particular, the distribution of the partition function $z_{N, 1}(n)=\sum_{\pi \in \Pi_{n, N}^{1}} w t(\pi)$ is the marginal distribution of $\mu_{n}^{N}(d y)$ on the first coordinate $y_{1}$, and hence uniquely characterized by (3.31).

(iv) The distribution of $P_{n, N}\left(d^{[1, n]}\right)$ is the measure in $d z$ given by

$$
\int \mu_{n}^{N}(d y) \bar{K}_{\theta}^{N}(y, d z) .
$$

(v) When $n=N$ we have the following simplification:

$$
\mu_{N}^{N}(d y)=\prod_{m=1}^{N} \prod_{i=1}^{N} \Gamma\left(\theta_{i}+\hat{\theta}_{m}\right)^{-1} e^{-y_{N}^{-1}} \Psi_{\theta}(y) \Psi_{\hat{\theta}}(y) \prod_{i=1}^{N} \frac{d y_{i}}{y_{i}} .
$$

In particular, for $s>0$,

$$
\int_{(0, \infty)^{N}} e^{-s y_{N}^{-1}} \mu_{N}^{N}(d y)=(1+s)^{-\sum_{i=1}^{N}\left(\theta_{i}+\hat{\theta}_{i}\right)}
$$

that is, the random variable $z_{N, N}(N)$ is inverse gamma distributed with parameter $\sum_{i=1}^{N}\left(\theta_{i}+\hat{\theta}_{i}\right)$.

The distribution of $z_{N, N}(N)$ can also be seen from (2.9).

Observe that the condition of $n \geq N$ is not restrictive when it comes to computing the Laplace transform in part (ii) of the above theorem. Indeed, if one wishes to compute the Laplace transform for $n<N$ then it suffices to transpose the parameter matrix and switch the role of $n$ and $N$. The distribution of the coordinate $y_{1}$ is unchanged by this procedure, and now the above corollary applies.

3.4. Pitman's $2 M-X$ theorem. Theorem 3.7 can be regarded as a variant of Pitman's ' $2 M-X$ theorem', which states that, if $X_{t}$ is a standard one-dimensional Brownian motion and $M_{t}=\max _{s \leq t} X_{s}$, then $2 M_{t}-X_{t}$ is a three-dimensional Bessel process. This theorem has vast generalizations $[6,10,11,15,25,37,41,44,45$, $46,47,51]$, many of which have been obtained via various analogues the 'Burke property' discussed in Remark 3.11 below. All of these can be regarded as variations of the statement that the stochastic evolution of the shape of the tableaux, obtained when applying variants of the RSK algorithm to random input data, has the Markov property. The first 'geometric' or 'positive temperature' version of this statement was discovered 
by Matsumoto and Yor [41], who showed that, for $X_{t}$ as above, the process $\log \int_{0}^{t} e^{2 X_{s}-X_{t}} d s, t>0$ is a diffusion on $\mathbb{R}$ with infinitesimal generator given by

$$
\frac{1}{2} \frac{d^{2}}{d x^{2}}+\left(\frac{d}{d x} \log K_{0}\left(e^{-x}\right)\right) \frac{d}{d x}
$$

where $K_{0}$ is the Macdonald function (with index 0). A multi-dimensional version of this theorem of Matsumoto and Yor is given in [44], which can be regarded as a particular specialization (scaling limit) of the main result in the present paper. It is also proved via an intertwining relation and is closely related to the quantum Toda lattice. The corresponding directed polymer model is defined on the semi-lattice $\mathbb{Z} \times \mathbb{R}$. Both models feature the $G L(N, \mathbb{R})$-Whittaker functions in an essential way; the relation between them is analogous to the relation between the Gaussian and Laguerre unitary ensembles in random matrix theory.

3.5. Invariant distributions. The Markov process defined by row insertion with a solvable inverse gamma parameter matrix turns out to have nice invariant distributions. The $z$-array itself cannot have an invariant distribution: for example, $z_{1,1}(n)=d_{n, 1} \cdots d_{1,1} z_{1,1}(0)$ evolves as a multiplicative random walk. Instead, we look at ratios of $z$-values.

Fix $N \geq 1$. For an array $z \in \mathbb{T}_{N}$ define the array $\eta=\left(\eta_{k \ell}\right)_{1 \leq \ell<k \leq N}$ of ratios by

$$
\eta_{k, \ell}=\frac{z_{k, \ell}}{z_{k-1, \ell}}, \quad 1 \leq \ell<k \leq N .
$$

The Markov process $z(n)$ then defines another random process $\eta(n)=\left(\eta_{k \ell}(n)\right)_{1 \leq \ell<k \leq N}$ by $\eta_{k, \ell}(n)=$ $z_{k, \ell}(n) / z_{k-1, \ell}(n)$. Denote again diagonals by $\eta_{\ell}(n)=\left(\eta_{k \ell}(n)\right)_{\ell<k \leq N}$ for $1 \leq \ell<N$. This new process $\eta(n)$ will also be a Markov chain.

Theorem 3.10. Let $z(n)$ evolve on the space $\mathbb{T}_{N}$ according to the Markovian dynamics governed by a solvable inverse-gamma weight matrix with parameters $\gamma_{i, j}=\hat{\theta}_{i}+\theta_{j}$, as specified by the transition kernels in (3.5).

(a) The process $\eta(n)$ is a Markov chain in its own filtration.

(b) Let $1 \leq j<N$. Assume $\theta_{1}<\theta_{2}<\cdots<\theta_{j}<\min \left\{\theta_{j+1}, \ldots, \theta_{N}\right\}$. Then the process $\left(\eta_{1}(n), \ldots, \eta_{j}(n)\right)$ has an invariant distribution where the variables $\left\{\eta_{k \ell}: 1 \leq \ell \leq j, \ell<k \leq N\right\}$ are independent with marginal distributions $\eta_{k \ell} \sim \Gamma^{-1}\left(\theta_{k}-\theta_{\ell}\right)$. If the process is started with this distribution, then the following statement holds for all times $n \geq 1$ : the variables $\left\{\eta_{k \ell}(n): 1 \leq \ell \leq j, \ell<k \leq N\right\} \cup\left\{z_{N \ell}(m) / z_{N \ell}(m-1): 1 \leq m \leq\right.$ $n, 1 \leq \ell \leq j\}$ are independent with marginals $\eta_{k \ell}(n) \sim \Gamma^{-1}\left(\theta_{k}-\theta_{\ell}\right)$ and $z_{N \ell}(m) / z_{N \ell}(m-1) \sim \Gamma^{-1}\left(\hat{\theta}_{m}+\theta_{\ell}\right)$.

Remark 3.11. Theorem 3.10 is an extension of a result of [59] for the directed polymer with inverse-gamma weights. This could be called a 'Burke property' by analogy with the Burke theorem (also known as the 'output theorem') of M/M/1 queues. According to the Burke theorem, for a reversible queue the number of customers in the system at time $t$ is independent of the departure process up to time $t$. This notion has an analogy in models with random weight matrices, and it has been used in the past to derive exact limit shapes $[57,58]$ and fluctuation exponents $[4,5,18]$. In fact, it was this property that led us to investigate the solvability of geometric RSK for inverse-gamma weight matrices. The analogous Burke property was found earlier in the Brownian polymer model [50] and was used to derive fluctuation exponents for that model in [60].

Theorem 3.10 is proved via (2.4) that represents a transition of the Markov process $z(n)$ in terms of a sequence of geometric row insertions. For this purpose we reformulate the row insertion step in terms of the ratios. In Definition 2.1 with fixed $1 \leq \ell<N$ the inputs of the row insertion were $\xi=\left(\xi_{\ell}, \ldots, \xi_{N}\right)$ and $b=\left(b_{\ell}, \ldots, b_{N}\right)$, and the outputs $\xi^{\prime}=\left(\xi_{\ell}^{\prime}, \ldots, \xi_{N}^{\prime}\right)$ and $b^{\prime}=\left(b_{\ell+1}^{\prime}, \ldots, b_{N}^{\prime}\right)$. Define now $\eta_{k}=\xi_{k} / \xi_{k-1}$ and $\eta_{k}^{\prime}=\xi_{k}^{\prime} / \xi_{k-1}^{\prime}$ for $\ell<k \leq N$, and also auxiliary variables $\zeta_{k}=\xi_{k}^{\prime} / \xi_{k}$ for $\ell \leq k \leq N$. The words are $\eta=\left(\eta_{\ell+1}, \ldots, \eta_{N}\right)$ and $\eta^{\prime}=\left(\eta_{\ell+1}^{\prime}, \ldots, \eta_{N}^{\prime}\right)$.

Lemma 3.12. Fix integers $1 \leq \ell \leq N$. In terms of the new variables, geometric row insertion transforms $(\eta, b)$ into $\left(\eta^{\prime}, b^{\prime}\right)$ via the following equations. Set first $\zeta_{\ell}=b_{\ell}$, and then inductively for $k=\ell+1, \ldots, N$ :

$$
\eta_{k}^{\prime}=b_{k}\left(1+\frac{\eta_{k}}{\zeta_{k-1}}\right), \quad \zeta_{k}=b_{k}\left(1+\frac{\zeta_{k-1}}{\eta_{k}}\right), \quad \text { and } \quad b_{k}^{\prime}=\left(\frac{1}{\zeta_{k-1}}+\frac{1}{\eta_{k}}\right)^{-1} .
$$

Next the row insertion step with random input. 
Lemma 3.13. Fix integers $1 \leq \ell<N$. Let $\alpha_{\ell+1}, \ldots, \alpha_{N}, \beta_{\ell}, \ldots, \beta_{N}$ be positive reals that satisfy $\beta_{k}=$ $\beta_{\ell}+\alpha_{k}$ for $\ell<k \leq N$. Assume that the random variables $\left\{\eta_{k}: \ell<k \leq N\right\} \cup\left\{b_{k}: \ell \leq k \leq N\right\}$ are independent with marginal distributions $\eta_{k} \sim \Gamma^{-1}\left(\alpha_{k}\right)$ and $b_{k} \sim \Gamma^{-1}\left(\beta_{k}\right)$. Then the random variables $\left\{\eta_{k}^{\prime}, b_{k}^{\prime}: \ell<k \leq N\right\} \cup\left\{\zeta_{N}\right\}$ are also independent with marginal distributions $\eta_{k}^{\prime} \sim \Gamma^{-1}\left(\alpha_{k}\right), b_{k}^{\prime} \sim \Gamma^{-1}\left(\beta_{k}\right)$, and $\zeta_{N} \sim \Gamma^{-1}\left(\beta_{\ell}\right)$.

Proof. From the assumptions and by definition, $\zeta_{\ell}=b_{\ell} \sim \Gamma^{-1}\left(\beta_{\ell}\right)$ and this variable is independent of $\left\{\eta_{\ell+1}, \ldots, \eta_{N}, b_{\ell+1}, \ldots, b_{N}\right\}$. Use equations (3.32) to prove, inductively on $m=\ell+1, \ldots, N$, that random variables $\left\{\eta_{\ell+1}^{\prime}, \ldots, \eta_{m}^{\prime}, b_{\ell+1}^{\prime}, \ldots, b_{m}^{\prime}, \zeta_{m}\right\}$ are independent, independent of $\left\{\eta_{m+1}, \ldots, \eta_{N}, b_{m+1}, \ldots, b_{N}\right\}$, and their marginal distributions are $\eta_{k}^{\prime} \sim \Gamma^{-1}\left(\alpha_{k}\right), b_{k}^{\prime} \sim \Gamma^{-1}\left(\beta_{k}\right)$ and $\zeta_{m} \sim \Gamma^{-1}\left(\beta_{\ell}\right)$. An induction step is achieved by applying (3.32) to the triple $\left(\zeta_{m}, \eta_{m+1}, b_{m+1}\right)$ to produce the new triple $\left(\zeta_{m+1}, \eta_{m+1}^{\prime}, b_{m+1}^{\prime}\right)$. Note that the parameter of $\zeta_{m}$ does not change with $m$. The case $m=N$ gives the lemma.

Proof of Theorem 3.10. (a) That $\eta(n)$ is itself a Markov process follows from the fact that from (3.32) we can build autonomous equations for this evolution.

(b) It suffices to show that the last claim is preserved by a step of the evolution. Consider the time $n$ transition from state $\eta=\eta(n-1)$ to state $\eta^{\prime}=\eta(n)$. The input weights are $a_{1}=\left(a_{11}, \ldots, a_{N 1}\right)=d^{[n]}=$ $\left(d_{n, 1}, \ldots, d_{n, N}\right)$ with $d_{n, k} \sim \Gamma^{-1}\left(\gamma_{n, k}\right)$, and this also defines the first diagonal $a_{1}$ of the auxiliary array in Def. 2.2. Assume that the variables $\left\{\eta_{k \ell}: 1 \leq \ell \leq j, \ell<k \leq N\right\} \cup\left\{z_{N \ell}(m) / z_{N \ell}(m-1): 1 \leq m \leq n-1,1 \leq\right.$ $\ell \leq j\}$ are independent with marginals $\eta_{k \ell} \sim \Gamma^{-1}\left(\theta_{k}-\theta_{\ell}\right)$ and $z_{N \ell}(m) / z_{N \ell}(m-1) \sim \Gamma^{-1}\left(\hat{\theta}_{m}+\theta_{\ell}\right)$. Let $\zeta_{N \ell}=z_{N \ell}(n) / z_{N \ell}(n-1)$ denote ratios defined along the transition process.

We prove the following statement inductively over $\ell=1, \ldots, j$.

The variables $\left\{\eta_{1}^{\prime}, \ldots, \eta_{\ell}^{\prime}, a_{\ell+1}, \zeta_{N 1}, \ldots, \zeta_{N \ell}\right\}$ are independent and independent of

$\left\{\eta_{\ell+1}, \ldots, \eta_{j}\right\} \cup\left\{z_{N i}(m) / z_{N i}(m-1): 1 \leq m \leq n-1,1 \leq i \leq j\right\}$, and

they have marginals $\eta_{k i}^{\prime} \sim \Gamma^{-1}\left(\theta_{k}-\theta_{i}\right), a_{k, \ell+1} \sim \Gamma^{-1}\left(\hat{\theta}_{n}+\theta_{k}\right)$ and $\zeta_{N i} \sim \Gamma^{-1}\left(\hat{\theta}_{n}+\theta_{i}\right)$.

The case $\ell=j$ completes the proof.

In the first row insertion step apply Lemma 3.13 with $\ell=1$ and inputs $\eta_{1}=\left(\eta_{2,1}, \ldots, \eta_{N, 1}\right)$ and $b=$ $\left(a_{11}, \ldots, a_{N 1}\right)$. Now $\alpha_{k}=\theta_{k}-\theta_{1}$ and $\beta_{k}=\gamma_{n, k}=\hat{\theta}_{n}+\theta_{k}$. According to the lemma, the outputs $\eta_{1}^{\prime}=\left(\eta_{2,1}^{\prime}, \ldots, \eta_{N, 1}^{\prime}\right), b^{\prime}=a_{2}=\left(a_{22}, \ldots, a_{N 2}\right)$ and $\zeta_{N 1}$ are independent and they have the correct marginal distributions: $\eta_{k, 1}^{\prime} \sim \Gamma^{-1}\left(\theta_{k}-\theta_{1}\right), a_{k 2} \sim \Gamma^{-1}\left(\hat{\theta}_{n}+\theta_{k}\right)$ and $\zeta_{N 1} \sim \Gamma^{-1}\left(\hat{\theta}_{n}+\theta_{1}\right)$. This gives (3.33) for $\ell=1$.

In the general step, assuming (3.33) for $\ell-1$, Lemma 3.13 is applied to inputs $\eta_{\ell}$ and $a_{\ell}$, with $\alpha_{k}=\theta_{k}-\theta_{\ell}$ and $\beta_{k}=\gamma_{n, k}=\hat{\theta}_{n}+\theta_{k}$. The outputs $\eta_{\ell}^{\prime}, a_{\ell+1}, \zeta_{N, \ell}$ have the right properties and the validity of (3.33) is exteded to $\ell$.

\section{Degenerations to KNOWn Results}

We detail rescalings of the inverse-gamma polymer which recover known results.

4.1. Directed last passage percolation and the Laguerre Unitary Ensemble. Fix a solvable parameter matrix $\gamma=\left(\gamma_{i, j}>0: i \geq 1,1 \leq j \leq N\right)$ such that $\gamma_{i, j}=\hat{\theta}_{i}+\theta_{j}$. Consider a family (indexed by $\left.\varepsilon>0\right)$ of solvable inverse-gamma weight matrices $d^{\varepsilon}=\left(d_{i, j}^{\varepsilon}: i \geq 1,1 \leq j \leq N\right)$ such that the entries are independent random variables and $d_{i, j}^{\varepsilon} \sim \Gamma^{-1}\left(\varepsilon \gamma_{i, j}\right)$. Keeping track of the $\varepsilon$ write $z_{k, \ell}^{\varepsilon}(n)$ as the elements of $z^{\varepsilon}(n)-$ the image of the weight matrix $\left(d_{i, j}^{\varepsilon}: 1 \leq i \leq n, 1 \leq j \leq N\right)$ under the geometric RSK correspondence. Write $F^{\varepsilon}(n)=\left(F_{k, \ell}^{\varepsilon}(n): 1 \leq \ell \leq k \leq N\right)$ where $F_{k, \ell}^{\varepsilon}(n)=\varepsilon \log z_{k, \ell}^{\varepsilon}(n)$.

With respect to the same solvable parameter matrix consider a weight matrix $w=\left(w_{i, j}: i \geq 1,1 \leq j \leq N\right)$ such that the entries are independent random variables and $w_{i, j} \sim \operatorname{Exp}\left(\gamma_{i, j}\right)$ (an exponential random variable with rate $\gamma_{i, j}$, or equivalently mean $\left.\left(\gamma_{i, j}\right)^{-1}\right)$. The classical RSK correspondence maps the weight matrix $w$ to a pair of Young tableaux $(P, Q)$ and is defined analogously but with the $(+, \times)$ algebra replaced by $(\max ,+)$. We focus on the $P$-tableaux and writing it in terms of a Gelfand-Zetlin pattern (a triangular array with interlacing). Recall the notation for non-intersecting paths $\Pi_{n, k}^{\ell}$ from Section 2. The weight of 
an $\ell$-tuple $\pi$ of paths is now

$$
\tilde{w} t(\pi)=\sum_{r=1}^{\ell} \sum_{(i, j) \in \pi_{r}} w_{i, j} .
$$

Define an array $L(n)=\left\{L_{k, l}(n): 1 \leq k \leq N, 1 \leq l \leq k \wedge n\right\}$ by

$$
L_{k, 1}(n)+\cdots+L_{k, \ell}(n)=\max _{\pi \in \Pi_{n, k}^{\ell}} \tilde{w} t(\pi) .
$$

$L(n)$ is the Gelfand-Zetlin pattern version of the $P$-tableau of the image of $\left(w_{i, j}: 1 \leq i \leq n, 1 \leq j \leq N\right)$ under the RSK correspondence. As in the case of the geometric RSK correspondence, when $n<N$ it is necessary to leave some entries of $L(n)$ undefined, or populate them with singular values (see Remark 2.4).

Proposition 4.1. The $n$-indexed process $\left(F^{\varepsilon}(n)\right)_{n \geq 0}$ converges in law, as $\epsilon \rightarrow 0$, to the $n$-indexed process $(L(n))_{n \geq 0}$.

Proof. This hinges on two observations. The first is that as $\varepsilon$ goes to zero, $\varepsilon \log d_{i, j}^{\varepsilon}$ converges in distribution to $w_{i, j}$. Hence by independence the whole array $\left\{\varepsilon \log d_{i, j}^{\varepsilon}\right\}$ converges in distribution to $w$ and (by the continuous mapping theorem) the random vectors

$$
\left(\sum_{r=1}^{\ell} \sum_{(i, j) \in \pi_{r}} \varepsilon \log d_{i, j}^{\varepsilon}\right)_{\pi \in \Pi_{n, k}^{\ell}, 1 \leq \ell \leq k \leq N} \Longrightarrow\left(\sum_{r=1}^{\ell} \sum_{(i, j) \in \pi_{r}} w_{i, j}\right)_{\pi \in \Pi_{n, k}^{\ell}, 1 \leq \ell \leq k \leq N} .
$$

The second observation is that on $\mathbb{R}^{m}$, the function $f_{\varepsilon}(x)=\varepsilon \log \sum_{i=1}^{m} e^{x_{i} / \varepsilon}$ converges uniformly, as $\varepsilon \rightarrow 0$, to the function $f_{0}(x)=\max \left(x_{i}: 1 \leq i \leq m\right)$. The process $\left(F^{\varepsilon}(n)\right)_{n>0}$ is formed by applying an array of functions of the type $f_{\varepsilon}$ to the elements of the vector on the left of (4.3). Combining the uniform convergence of functions of this type with the convergence in distribution in (4.3), the claimed convergence of the processes follows.

It is worth noting that in the above limit we only recover the directed last passage percolation model with exponentially distributed weights, and not the model with geometric distribution studied in [33]. Since our log-gamma distributions are continuous, it does not seem possible to recover the discrete geometric distribution (but rather just their continuous exponential counterparts).

Let us now briefly recall the connections between bottom row of $L(n)$ and the eigenvalues of the Laguerre Unitary Ensemble (LUE). Consider an array $\left(A_{i, j}: 1 \leq i \leq N, j \geq 1\right)$ of independent complex zero-mean Gaussian distributed random variables with variance $\left(\gamma_{j, i}\right)^{-1}$. We have changed the order of $i$ and $j$ since we now set $A(n)=\left(A_{i, j}: 1 \leq i \leq N, 1 \leq j \leq n\right)$ and treat $A(n)$ as a matrix with (row,col) notation. Set $M(n)=A(n) A(n)^{*}$ the $N \times N$ generalized Wishart random matrix, and define for each $n$, a vector of ordered (largest to smallest) eigenvalues of $M(n): \lambda(n)=\left(\lambda_{1}(n), \ldots, \lambda_{N}(n)\right)$.

When $\gamma_{i, j}=1$ for all $i, j$, Johansson [33] showed that for fixed $n, \lambda_{1}(n) \stackrel{(d)}{=} L_{N, 1}(n)$. This was strengthened by [24] to show that for the same parameters as in [33] and for $n \geq N$ fixed, the vector $\left(\lambda_{i}(n)\right)_{i=1}^{N} \stackrel{(d)}{=}$ $\left(L_{N, i}(n)\right)_{i=1}^{N}$. In [22, 27] equality in law was shown for the processes $\left(\lambda_{1}(n)\right)_{n \geq 1}$ and $\left(L_{N, 1}(n)\right)_{n \geq 1}$.

Turning to the general case for the parameters $\gamma_{i, j}$, [14] proved that for $n$ fixed, $\lambda_{1}(n) \stackrel{(d)}{=} L_{N, 1}(n)$ and conjectured equality of the corresponding processes in $n$. This was then proved in [23], whose methods (combining Theorem 3.1 and Lemma 4.1) show equality in law of the process $(\lambda(n))_{n \geq 1}$ and $\left(L_{N}(n)\right)_{n \geq 1}$ where $L_{N}(n)=\left(L_{N, i}(n)\right)_{i=1}^{N}$ and where only non-zero eigenvalues/non-singular entries of $L$ are considered.

Combining Proposition 4.1 with the above discussion one sees that the logarithm of the bottom row of the image of a solvable weight matrix under the geometric RSK correspondence are analogous to the eigenvalues of the LUE ensemble. This connection can be seen from the integral formulas we have derived in (i) of Theorem 3.9. We perform point-wise asymptotics to demonstrate this connection we have proved above. Performing the change of variables to that formula given by $y_{i} \mapsto e^{\varepsilon^{-1} x_{i}}, \theta \mapsto \varepsilon \theta$ and $\lambda \mapsto \varepsilon \lambda$ we find that the measure for $\left(F_{N, \ell}^{\varepsilon}(n)\right)_{\ell=1}^{N}=\left(x_{1}, \ldots, x_{N}\right)$ is given by 


$$
\prod_{i=1}^{N} \varepsilon^{-1} d x_{i} \Psi_{\varepsilon \theta}^{N}\left(e^{\varepsilon^{-1} x}\right) \int_{\iota \mathbb{R}^{N}} \varepsilon^{N} d \lambda \Psi_{\varepsilon(-\lambda)}^{N}\left(e^{\varepsilon^{-1} x}\right) \prod_{m=1}^{n} \prod_{i=1}^{N} \frac{\Gamma\left(\varepsilon\left(\hat{\theta}_{m}+\lambda_{i}\right)\right)}{\Gamma\left(\varepsilon\left(\theta_{i}+\hat{\theta}_{m}\right)\right)} s_{N}(\varepsilon \lambda) .
$$

We may now evaluate the $\varepsilon \rightarrow 0$ asymptotics: For the Gamma functions we employ the expansion near zero; for the measure $s_{N}$ we employ the Euler Gamma reflection formula; for the Whittaker functions we can write the Whittaker functions in additive variables (and perform a sign change) $\psi_{\lambda}^{N}(x)=\Psi_{-\lambda}^{N}\left(e^{x}\right)$, and then use the fact (see for instance $[35,44]$ ) to see

$$
\lim _{\varepsilon \rightarrow 0} \varepsilon^{\frac{N^{2}-N}{2}} \psi_{\varepsilon \lambda}^{N}\left(\varepsilon^{-1} x\right)=\tilde{\psi}_{\lambda}^{N}(x)=\tilde{\psi}_{\lambda_{1}, \ldots, \lambda_{N}}^{N}(x)=(-1)^{\frac{N^{2}-N}{2}} \frac{\operatorname{det}\left(e^{\lambda_{i} x_{j}}\right)_{i, j=1}^{N}}{h(\lambda)}
$$

where $h(\lambda)=\prod_{1 \leq i<j \leq N}\left(\lambda_{j}-\lambda_{i}\right)$ is the Vandermonde determinant.

The expansion of the Gamma function near zero shows that as $\varepsilon$ goes to zero,

$$
\prod_{m=1}^{n} \prod_{i=1}^{N} \frac{\Gamma\left(\varepsilon\left(\hat{\theta}_{m}+\lambda_{i}\right)\right)}{\Gamma\left(\varepsilon\left(\theta_{i}+\hat{\theta}_{m}\right)\right)} \rightarrow \prod_{m=1}^{n} \prod_{i=1}^{N} \frac{\theta_{i}+\hat{\theta}_{m}}{\hat{\theta}_{m}+\lambda_{i}}
$$

Likewise, the Euler Gamma reflection formula and the fact that $\sin (\pi x) /(\pi x) \rightarrow 1$ as $x \rightarrow 0$ yields

$$
\varepsilon^{-N^{2}+N} s_{N}(\varepsilon \lambda) \rightarrow \frac{1}{(2 \pi \iota)^{N} N !}(-1)^{\frac{N^{2}-N}{2}} h(\lambda)^{2},
$$

where $h(\lambda)=\prod_{1 \leq i<j \leq N}\left(\lambda_{j}-\lambda_{i}\right)$ is the Vandermonde determinant.

Putting these asymptotics together we have that the limit of our measure is given by

$$
\prod_{i=1}^{N} d x_{i} \frac{\operatorname{det}\left(e^{-\theta_{i} x_{j}}\right)_{i, j=1}^{N}}{h(\theta)} \frac{1}{(2 \pi i)^{N} N !} \int_{\iota \mathbb{R}^{N}} d \lambda \operatorname{det}\left(e^{\lambda_{i} x_{j}}\right)_{i, j=1}^{N} h(\lambda) \prod_{m=1}^{n} \prod_{i=1}^{N} \frac{\theta_{i}+\hat{\theta}_{m}}{\hat{\theta}_{m}+\lambda_{i}} .
$$

For simplicity let us assume that $N=n$. Then this can be rewritten as

$$
\frac{1}{Z_{N, N}} \operatorname{det}\left(e^{-\theta_{i} x_{j}}\right)_{i, j=1}^{N} D(x, \hat{\theta}) \prod_{i=1}^{N} d x_{i}
$$

where we have

$$
D(x, \hat{\theta})=\frac{1}{(2 \pi i)^{N} N !} \int d \lambda \operatorname{det}\left(e^{\lambda_{i} x_{j}}\right)_{i, j=1}^{N} h(\hat{\theta}) h(\lambda) \prod_{m=1}^{N} \prod_{i=1}^{N} \frac{1}{\hat{\theta}_{m}+\lambda_{i}},
$$

where the integrals are along lines parallel to the imaginary axis and to the right of the poles, and where

$$
Z_{N, N}=\frac{h(\theta) h(\hat{\theta})}{\prod_{i, m=1}^{N}\left(\theta_{i}+\hat{\theta}_{m}\right)}=\operatorname{det}\left(\frac{1}{\theta_{i}+\hat{\theta}_{j}}\right)_{i, j=1}^{N} .
$$

An application of the Residue Theorem provides that

$$
D(x, \hat{\theta})=\operatorname{det}\left(e^{-\hat{\theta}_{i} x_{j}}\right)_{i, j=1}^{N} \prod_{i=1}^{N} \mathbf{1}_{x_{i} \geq 0}
$$

and hence our measure is simply

$$
\frac{1}{Z_{N, N}} \operatorname{det}\left(e^{-\theta_{i} x_{j}}\right)_{i, j=1}^{N} \operatorname{det}\left(e^{-\hat{\theta}_{i} x_{j}}\right)_{i, j=1}^{N} \prod_{i=1}^{N} d x_{i} \mathbf{1}_{x_{i} \geq 0}
$$

which coincides exactly with the formula given directly for last passage percolation and for the generalized Wishart ensemble in [14]. 
4.2. Semi-discrete directed polymer in a Brownian environment. We will now indicate how an appropriate scaling of the solvable parameter matrix $\left(\gamma_{i, j}\right)$ can be used to recover the semi-discrete directed polymer in a Brownian environment studied in [50,42,60,44], as a scaling limit of $z_{N 1}(n)$. In particular, we will consider the weight matrix $\left(d_{i j}\right)$ with solvable parameter matrix $\gamma_{i j}=n$ and we will let $n$ tend to infinity. We would first need some facts about the digamma and the trigamma functions, which are defined as

$$
\Psi_{0}(\theta)=\frac{d}{d \theta} \log \Gamma(\theta) \quad \text { and } \quad \Psi_{1}(\theta)=\frac{d^{2}}{d \theta^{2}} \log \Gamma(\theta) .
$$

In particular, we have that for $\theta$ large

$$
\Psi_{0}(\theta)=\log \theta-\frac{1}{2 \theta}+o\left(\frac{1}{\theta}\right), \quad \Psi_{1}(\theta)=\frac{1}{\theta}+o\left(\frac{1}{\theta}\right) .
$$

We also notice that for an inverse Gamma random variable $d$ with parameter $\theta$ it holds that

$$
\Psi_{0}(\theta)=-E[\log d] \text { and } \Psi_{1}(\theta)=\operatorname{Var}(\log d) .
$$

We write $\log z_{N 1}(n)$ in terms of the weights $d_{i j}$ (iid inverse Gamma random variables with parameter $n$ ) as

$$
\begin{aligned}
\log z_{N 1}(n)= & \log \sum_{1 \leq i_{1} \cdots \leq i_{N}=n} \exp \left[\sum_{j=1}^{N} \sum_{i_{j-1} \leq i \leq i_{j}} \log d_{i j}\right] \\
= & \log n^{-(N-1)} \sum_{\substack{1 \leq i_{1} \cdots \leq i_{N}=n\\
}} \exp \left[\sqrt{n \Psi_{1}(n)} \sum_{j=1}^{N} \frac{1}{\sqrt{n}} \sum_{i_{j-1} \leq i \leq i_{j}} \frac{\log d_{i j}+\Psi_{0}(n)}{\sqrt{\Psi_{1}(n)}}\right] \\
& +(N-1) \log n-(n+N-1) \Psi_{0}(n) .
\end{aligned}
$$

Using (4.5) we have that $n \Psi_{1}(n) \rightarrow 1$ and $(N-1) \log n-(n+N-1) \Psi_{0}(n)=-n \log n+\frac{1}{2}+o(1)$, as $n$ tends to infinity. It is now an easy consequence of Donsker's invariance principle and the Riemann integral definition that

$$
\log \left(n^{n} z_{N 1}(n)\right)-\frac{1}{2} \Longrightarrow \log \int \cdots \int_{0 \leq t_{1} \leq \cdots \leq t_{n-1} \leq 1} e^{\sum_{i=1}^{N}\left(B^{i}\left(t_{i}\right)-B^{i}\left(t_{i-1}\right)\right)} d t_{1} \cdots d t_{n-1},
$$

for $n$ tending to infinity, with $B^{i}(\cdot)$ independent, standard Brownian motions. This is the directed polymer model studied in $[50,42,60,44]$. Along the same lines it follows that the whole pattern $\left(z_{k \ell}(n)\right)$ obeys similar scaling limits.

\section{Proof of MAIN Results}

5.1. Proof of Proposition 3.4. We prove the intertwining relation (3.13) in two steps. We use induction on $N$ to show that (3.8) is valid. The inductive step will also reveal an inductive property of the eigenfunctions $w_{\theta}^{N}$.

The case $N=1$ in (3.8) is immediate since $\Pi_{\gamma^{[n]}}^{1}=P_{\gamma^{[n]}}^{1}$ and $K_{\theta_{1}}^{1}$ is the identity operator.

A supporting step of the proof is an intertwining that involves only two rows of the array $z$. For this purpose introduce two further kernels for $2 \leq k \leq N \leq n$ : a time $n$ kernel $R_{\gamma^{[n]}}^{k}$ on $\mathbb{Y}_{k-1} \times \mathbb{Y}_{k}$ by

$$
R_{\gamma^{[n]}}^{k}\left(\left(z^{k-1}, z^{k}\right), d \tilde{z}^{k-1}, d \tilde{z}^{k}\right)=P_{\gamma^{[n]}}^{k-1}\left(z^{k-1}, d \tilde{z}^{k-1}\right) L_{\gamma_{n, k}}^{k}\left(\left(z^{k-1}, z^{k} ; \tilde{z}^{k-1}\right), d \tilde{z}^{k}\right)
$$

and a time-homogeneous kernel from $\mathbb{Y}_{k}$ to $\mathbb{Y}_{k-1}$ by

$$
\Lambda_{\theta}^{k}(y, d x)=\left\{\prod_{\ell=1}^{k-1}\left(\frac{x_{\ell}}{y_{\ell}}\right)^{\theta_{k}-\theta_{\ell}}\right\} \exp \left[-\sum_{\ell=1}^{k-1}\left(\frac{x_{\ell}}{y_{\ell}}+\frac{y_{\ell+1}}{x_{\ell}}\right)\right] \prod_{\ell=1}^{k-1} \frac{d x_{\ell}}{x_{\ell}} .
$$

Lemma 5.1. At every time $n \geq 1$ and for all $y \in \mathbb{Y}_{N}$, we have the following equality of measures on $\mathbb{Y}_{N-1} \times \mathbb{Y}_{N}$, in terms integration variables $\left(d z^{N-1}, d z^{N}\right)$ :

$$
P_{\gamma^{[n]}}^{N}\left(y, d z^{N}\right) \Lambda_{\theta}^{N}\left(z^{N}, d z^{N-1}\right)=\int_{\hat{x} \in \mathbb{R}_{+}^{N-1}} \Lambda_{\theta}^{N}(y, d \hat{x}) R_{\gamma^{[n]}}^{N}\left((\hat{x}, y), d z^{N-1}, d z^{N}\right)
$$


With (5.2) we can give this alternative representation to the intertwining kernel (3.7) from $\mathbb{Y}_{N}$ to $\mathbb{T}_{N}$ :

$$
K_{\theta}^{N}(y, d z)=\delta_{y}\left(d z^{N}\right) \prod_{j=0}^{N-2} \Lambda_{\theta}^{N-j}\left(z^{N-j}, d z^{N-j-1}\right)
$$

Postpone the proof of Lemma 5.1 for a moment. With (5.4) and Lemma 5.1 we can complete the proof of Proposition 3.4 by checking the induction step. Assume (3.8) for $N-1$.

$$
\int_{\tilde{y} \in \mathbb{R}_{+}^{N}} P_{\gamma^{[n]}}^{N}(y, d \tilde{y}) K_{\theta}^{N}\left(\tilde{y}, d z^{1, N}\right)=P_{\gamma^{[n]}}^{N}\left(y, d z^{N}\right) \prod_{j=0}^{N-2} \Lambda_{\theta}^{N-j}\left(z^{N-j}, d z^{N-j-1}\right)
$$

by Lemma 5.1

$$
=\int_{\hat{x} \in \mathbb{R}_{+}^{N-1}} \Lambda_{\theta}^{N}(y, d \hat{x}) R_{\gamma}^{N[n]}\left((\hat{x}, y), d z^{N-1}, d z^{N}\right) \prod_{j=1}^{N-2} \Lambda_{\theta}^{N-j}\left(z^{N-j}, d z^{N-j-1}\right)
$$

by definition of $R_{\gamma[n]}^{N}$

$$
\begin{gathered}
=\int_{\hat{x} \in \mathbb{R}_{+}^{N-1}} \Lambda_{\theta}^{N}(y, d \hat{x}) P_{\gamma}^{N-1}\left(\hat{x}, d z^{N-1}\right) \prod_{j=1}^{N-1} \Lambda_{\theta}^{N-j}\left(z^{N-j}, d z^{N-j-1}\right) \\
\times L_{\gamma_{n, N}}^{N}\left(\left(\hat{x}, y ; z^{N-1}\right), d z^{N}\right)
\end{gathered}
$$

by definition of $K_{\theta_{1: N-1}^{N-1}}^{N-1}$ and the induction assumption

$$
\begin{gathered}
=\int_{\hat{x} \in \mathbb{R}_{+}^{N-1}} \Lambda_{\theta}^{N}(y, d \hat{x}) \int_{\tilde{z}^{1, N-1} \in T_{N-1}} K_{\theta}^{N-1}\left(\hat{x}, d \tilde{z}^{1, N-1}\right) \Pi_{\gamma^{[n]}}^{N-1}\left(\tilde{z}^{1, N-1}, d z^{1, N-1}\right) \\
\times L_{\gamma_{n, N}}^{N}\left(\left(\hat{x}, y ; z^{N-1}\right), d z^{N}\right)
\end{gathered}
$$

by noting that $\tilde{z}^{N-1}=\hat{x}$ under $K_{\theta_{1: N-1}^{N-1}}^{N}\left(\hat{x}, d \tilde{z}^{1, N-1}\right)$, and by definition of $\Pi_{\gamma^{[n]}}^{N}$

$$
\begin{aligned}
& =\int_{\hat{x} \in \mathbb{R}_{+}^{N-1}} \Lambda_{\theta}^{N}(y, d \hat{x}) \int_{\tilde{z}^{1, N-1} \in T_{N-1}} K_{\theta}^{N-1}\left(\hat{x}, d \tilde{z}^{1, N-1}\right) \Pi_{\gamma}^{N[n]}\left(\left(\tilde{z}^{1, N-1}, y\right), d z^{1, N}\right) \\
& =\int_{\tilde{z}^{1, N} \in T_{N}} K_{\theta}^{N}\left(y, d \tilde{z}^{1, N}\right) \Pi_{\gamma^{[n]}}^{N}\left(\tilde{z}^{1, N}, d z^{1, N}\right) .
\end{aligned}
$$

This checks (3.8) for $N$.

Proof of Lemma 5.1. Take a test function $g$ on $\mathbb{Y}_{N-1} \times \mathbb{Y}_{N}$, and collect and rearrange all the factors in the integral against the kernel on the right-hand side of (5.3):

$$
\begin{aligned}
& \int_{\mathbb{R}_{+}^{N-1}} \Lambda_{\theta}^{N}(y, d \hat{x}) \int_{\mathbb{R}_{+}^{N-1} \times \mathbb{R}_{+}^{N}} R_{\gamma}^{N[n]}((\hat{x}, y), d \tilde{x}, d \tilde{y}) g(\tilde{x}, \tilde{y}) \\
& =\left\{\prod_{j=1}^{N} \Gamma\left(\gamma_{n, j}\right)^{-1}\right\} \int_{\mathbb{R}_{+}^{N-1} \times \mathbb{R}_{+}} d \tilde{x} d \tilde{y}_{1} \int_{\mathbb{R}_{+}^{N-1}} d \hat{x} \\
& \quad \times \exp \left[-\sum_{\ell=1}^{N-1}\left(\frac{\hat{x}_{\ell}}{y_{\ell}}+\frac{y_{\ell+1}}{\hat{x}_{\ell}}+\frac{\hat{x}_{\ell}}{\tilde{x}_{\ell}}\right)-\sum_{\ell=1}^{N-2} \frac{\tilde{x}_{\ell+1}}{\hat{x}_{\ell}}-\frac{y_{1}+\tilde{x}_{1}}{\tilde{y}_{1}}\right] \\
& \quad \times\left(y_{1}+\tilde{x}_{1}\right)^{\gamma_{n}, N} \tilde{y}_{1}^{-\gamma_{n, N}-1} \cdot \prod_{i=1}^{N-1}\left(\frac{\hat{x}_{i}}{y_{i}}\right)^{\theta_{N}-\theta_{i}} \cdot \prod_{j=1}^{N-1}\left(\hat{x}_{j}^{\gamma_{n, j}-1} \tilde{x}_{j}^{-\gamma_{n, j}-1}\right) \\
& \quad \times g\left(\tilde{x}, \tilde{y}_{1},\left\{\frac{y_{\ell-1} \tilde{x}_{\ell-1}}{\hat{x}_{\ell-1}} \cdot \frac{y_{\ell}+\tilde{x}_{\ell}}{y_{\ell-1}+\tilde{x}_{\ell-1}}\right\}_{2 \leq \ell \leq N-1}, \frac{y_{N} y_{N-1} \tilde{x}_{N-1}}{\hat{x}_{N-1}\left(y_{N-1}+\tilde{x}_{N-1}\right)}\right) .
\end{aligned}
$$


Change variables in the inner integral from $\hat{x}$ to $\tilde{y}_{2, N}$ so that the $g$-integrand becomes simply $g(\tilde{x}, \tilde{y})$. Recall that $\gamma_{n, j}=\theta_{j}+\hat{\theta}_{n}$. After matching up all the powers of the variables the integral above acquires the form

$$
\begin{gathered}
\left\{\prod_{j=1}^{N} \Gamma\left(\gamma_{n, j}\right)^{-1}\right\} \int_{\mathbb{R}_{+}^{N-1} \times \mathbb{R}_{+}^{N}} d \tilde{x} d \tilde{y} \exp \left[-\sum_{\ell=1}^{N-1}\left(\frac{\tilde{y}_{\ell+1}}{y_{\ell}}+\frac{\tilde{y}_{\ell+1}}{\tilde{x}_{\ell}}+\frac{\tilde{x}_{\ell}}{\tilde{y}_{\ell}}\right)-\sum_{\ell=1}^{N} \frac{y_{\ell}}{\tilde{y}_{\ell}}\right] \\
\times \prod_{j=1}^{N-1} \tilde{x}_{j}^{\theta_{N}-\theta_{j}-1} \cdot \prod_{j=1}^{N}\left(y_{j}^{\gamma_{n, j}} \tilde{y}_{j}^{-\gamma_{n, N}-1}\right) \cdot g(\tilde{x}, \tilde{y}) .
\end{gathered}
$$

That this agrees with the integral coming from the left-hand side of (5.3) is just a matter of substituting in the explicit formulas of the kernels. The proof of Lemma 5.1 is complete.

5.2. Proof of Theorem 3.7. Parts (i) and (ii) follow immediately from consequences (ii) and (i) (respectively) of Proposition 3.3 when applied to $\bar{P}_{\gamma[n]}^{N}, \bar{K}_{\theta}^{N}$, and $\Pi_{\gamma[n]}^{N}$. In order to apply Proposition 3.3 we must check two conditions. Condition (1) follows from Corollary 3.6 and condition (2) follows from the definition (3.12) of $\bar{K}_{\theta}^{N}$.

Part (iii) follows from (3.26) setting $f(z)=\prod_{i=1}^{N} x_{i}(z)^{-\lambda_{i}}$ via the use of relations (3.14), (3.15), (3.22), $(3.23)$ :

$$
\begin{aligned}
\frac{1}{\prod_{i=1}^{N} y_{i}^{-\theta_{i}} w_{\theta}^{N}(y)} \int_{\mathbb{T}_{N}} \prod_{i=1}^{N} x_{i}(z)^{-\lambda_{i}} M_{\theta}^{N}(y, d z) & =\frac{1}{\Psi_{\theta}^{N}(y)} \int_{\mathbb{T}_{N}} \prod_{i=1}^{N} x_{i}(z)^{-\theta_{i}-\lambda_{i}} M^{N}(y, d z) \\
& =\frac{\Psi_{\theta+\lambda}^{N}(y)}{\Psi_{\theta}^{N}(y)}
\end{aligned}
$$

In fact, if the $\lambda_{i}$ have non-zero real part then $f$ is not bounded. However if we set $f_{n}(x)=f(x) \mathbf{1}_{n^{-1} \leq x \leq n}$ then the claimed formula follows from dominated convergence and the fact that $\int_{\mathbb{T}_{N}} \bar{K}_{\theta}^{N}(y, d z)|f(z)|$ is bounded.

Part (iv), equation (3.27) follows by integrating equation (3.26) over $\mu_{n}^{N}\left(y^{0}, d y\right)$ or using the intertwining relation (3.8) directly, as discussed in Remark 3.8.

To deduce (3.28) we use the Plancherel formula (3.19). Let $\mu_{n}^{N}\left(y^{0}, y\right)$ denote the density of $\mu_{n}^{N}\left(y^{0}, d y\right)$. It exists because $\mu_{n}^{N}\left(y^{0}, d y\right)$ comes from composing kernels with densities. Multiply both sides of (3.27) by $\int_{(0, \infty)} f(y) \Psi_{\theta}^{N}(y) \Psi_{-\lambda}^{N}(y) \prod_{i} y_{i}{ }^{-1} d y_{i}$, integrate over $\iota \mathbb{R}^{N}$ with respect to $s_{N}(\lambda) d \lambda$ and use (3.19). The application of the Plancherel identity is valid since $f(y) \Psi_{\theta}^{N}(y)$ is in $L^{2}\left((0, \infty)^{N}, \prod_{i} d y_{i} / y_{i}\right)$ for $f$ continuous and compactly supported on $(0, \infty)^{N}$, and by the next lemma.

Lemma 5.2. Let $\gamma_{i, j}=\hat{\theta}_{i}+\theta_{j}>0$ be a solvable parameter matrix. Assume $\theta_{j}-\hat{\theta}_{i}<0<\hat{\theta}_{i}$ for all $i, j \geq 1$. Then for all $y^{0} \in(0, \infty)^{N}$ and $n \geq 1$, the function

$$
\frac{\mu_{n}^{N}\left(y^{0}, y\right)}{\Psi_{\theta}^{N}(y)} \prod_{i=1}^{N} y_{i}
$$

is in $L^{2}\left((0, \infty)^{N}, \prod_{i} d y_{i} / y_{i}\right)$.

Proof. Iterating definition (3.11) and using (3.15) gives

$$
\frac{\mu_{n}^{N}\left(y^{0}, y\right)}{\Psi_{\theta}^{N}(y)}=\left(\prod_{j=1}^{N} y_{j}^{\theta_{j}}\right) \frac{p_{n}^{N}\left(y^{0}, y\right)}{w_{\theta}^{N}\left(y^{0}\right)}
$$

where $p_{n}^{N}\left(y^{0}, y\right)$ is the density of the $n$-fold composition of kernels $P_{\gamma^{[1]}}^{N}\left(y^{0}, d y^{1}\right), \ldots, P_{\gamma^{[n]}}^{N}\left(y^{n-1}, d y\right)$ from (3.6). Let

$$
r_{\alpha}(u, v)=\Gamma(\alpha)^{-1}\left(\frac{u}{v}\right)^{\alpha} \frac{e^{-u / v}}{v}, \quad u, v \in(0, \infty),
$$

denote the transition density of a multiplicative random walk on $(0, \infty)$ with $\Gamma^{-1}(\alpha)$-distributed steps, and let

$$
R_{n, j}\left(u^{0}, u^{n}\right)=\int_{(0, \infty)^{n-1}} \prod_{i=1}^{n} r_{\gamma_{i, j}}\left(u^{i-1}, u^{i}\right) d u^{n-1} \cdots d u^{1}
$$


denote the $n$-step transition density with parameters $\gamma_{i, j}$ from column $j$ of our solvable matrix. By dropping the killing term from (3.6) and by an application of Jensen's inequality,

$$
\left(p_{n}^{N}\left(y^{0}, y\right)\right)^{2} \leq \prod_{j=1}^{N}\left(R_{n, j}\left(y_{j}^{0}, y_{j}\right)\right)^{2} \leq \prod_{j=1}^{N} \int_{0}^{\infty} R_{n-1, j}\left(y_{j}^{0}, \tilde{y}_{j}\right)\left(r_{\gamma_{n, j}}\left(\tilde{y}_{j}, y_{j}\right)\right)^{2} d \tilde{y}_{j} .
$$

Put these together, noting that the integrals factor over $(0, \infty)^{N}$ :

$$
\begin{aligned}
\int_{(0, \infty)^{N}} & \left(\prod_{j=1}^{N} y_{j}\right)\left(\frac{\mu_{n}^{N}\left(y^{0}, y\right)}{\Psi_{\theta}^{N}(y)}\right)^{2} d y \\
\leq & \frac{1}{w_{\theta}^{N}\left(y^{0}\right)^{2}} \prod_{j=1}^{N} \int_{(0, \infty)^{2}} y_{j}^{2 \theta_{j}+1} R_{n-1, j}\left(y_{j}^{0}, \tilde{y}_{j}\right)\left(r_{\gamma_{n, j}}\left(\tilde{y}_{j}, y_{j}\right)\right)^{2} d y_{j} d \tilde{y}_{j} \\
= & \frac{C_{N, n}\left(\hat{\theta}^{[n]}, \theta\right)}{w_{\theta}^{N}\left(y^{0}\right)^{2}} \prod_{j=1}^{N} \int_{(0, \infty)} \tilde{y}_{j}^{2 \theta_{j}} R_{n-1, j}\left(y_{j}^{0}, \tilde{y}_{j}\right) d \tilde{y}_{j} \\
= & \frac{C_{N, n}\left(\hat{\theta}^{[n]}, \theta\right)}{\Psi_{\theta}^{N}\left(y^{0}\right)^{2}} \prod_{j=1}^{N} \prod_{i=1}^{n-1} \mathbb{E}\left[d_{i, j}^{2 \theta_{j}}\right]<\infty .
\end{aligned}
$$

The first equality above integrates away the variables $y_{j}$, and the finiteness of the constant $C_{N, n}\left(\hat{\theta}^{[n]}, \theta\right)$ depends on $\hat{\theta}_{n}>0$. The second equality uses the independence of random walk steps. The finiteness of the expectations is equivalent to $\theta_{j}-\hat{\theta}_{i}<0$.

5.3. Proof of Theorem 3.9. To prove part (i) set $y^{0}=y^{0, M}$ in (3.28). Asymptotic relation (20) in [44] gives that, as $M \rightarrow \infty$, the ratio $\Psi_{\lambda}^{N}\left(y^{0, M}\right) / \Psi_{\theta}^{N}\left(y^{0, M}\right) \rightarrow 1$. Therefore, we only need to demonstrate that the limit $M \rightarrow \infty$ can be passed inside the integral. This follows from dominated convergence since

$$
\left|\frac{\Psi_{\lambda}^{N}\left(y^{0, M}\right)}{\Psi_{\theta}^{N}\left(y^{0, M}\right)}\right| \leq\left|\frac{\Psi_{0}^{N}\left(y^{0, M}\right)}{\Psi_{\theta}^{N}\left(y^{0, M}\right)}\right|
$$

for $\lambda \in \iota \mathbb{R}^{N}$ and the fact that the rest of the integrand is in $L^{1}\left(\iota \mathbb{R}^{N}, s_{N}(\lambda) d \lambda\right)$. The latter follows from the fact that $\int_{(0, \infty)^{N}} f(y) \Psi_{\theta}(y) \Psi_{-\lambda}(y) \prod_{i} d y_{i} / y_{i} \in L^{2}\left(\iota \mathbb{R}^{N}, s_{N}(\lambda) d \lambda\right)$, by the Plancherel isomorphism and the fact that $f$ is bounded and compactly supported, and $\prod_{m} \prod_{i} \Gamma\left(\hat{\theta}_{m}+\lambda_{i}\right) / \Gamma\left(\theta_{i}+\hat{\theta}_{m}\right) \in L^{2}\left(\iota \mathbb{R}^{N}, s_{N}(\lambda) d \lambda\right)$, for $n \geq N$. Indeed, using the asymptotics

$$
\lim _{x_{2} \rightarrow \infty}\left|\Gamma\left(x_{1}+\iota x_{2}\right)\right| e^{\frac{\pi}{2}\left|x_{2}\right|}\left|x_{2}\right|^{\frac{1}{2}-x_{1}}=\sqrt{2 \pi}, \quad x_{1}, x_{2} \in \mathbb{R}
$$

it follows that

$$
\begin{aligned}
\left|\prod_{m=1}^{n} \prod_{i=1}^{N} \frac{\Gamma\left(\hat{\theta}_{m}+\lambda_{i}\right)}{\Gamma\left(\theta_{i}+\hat{\theta}_{m}\right)}\right|^{2} s_{N}(\lambda) & \sim e^{-\pi n \sum_{i=1}^{N}\left|\lambda_{i}\right|+\frac{\pi}{2} \sum_{1 \leq i \neq j \leq N}\left|\lambda_{i}-\lambda_{j}\right|} \\
& \lesssim e^{-\pi n \sum_{i=1}^{N}\left|\lambda_{i}\right|+\pi(N-1) \sum_{i=1}^{N}\left|\lambda_{i}\right|},
\end{aligned}
$$

which decays exponentially when $n \geq N$.

Part (ii) follows from the Whittaker integral identity (3.20)

$$
\int_{(0, \infty)^{N}} e^{-s y_{1}} \Psi_{\theta}(y) \Psi_{-\lambda}(y) \prod_{i} \frac{d y_{i}}{y_{i}}=s^{\sum\left(-\lambda_{i}+\theta_{j}\right)} \prod_{i, j} \Gamma\left(\lambda_{i}-\theta_{j}\right) \in L^{2}\left(\iota \mathbb{R}^{N}, s_{N}(\lambda) d \lambda\right) .
$$

We can now repeat the argument used in the proof of part (i), using the function $f(y)=e^{-s y_{1}}$ (instead of a compactly supported $f$ ).

To prove part (iii) we show in Proposition 5.3 below a more general statement: the entire array $z(n)$ converges in distribution, as $M \rightarrow \infty$, to the one defined by the path configurations, noting that this statement can make sense only for the portion of the array constructed by time $n$.

Let $P^{z}$ denote the probability distribution of the process $z(\cdot)$ when the initial state is $z(0)=z \in \mathbb{T}_{N}$, and let $E^{z}$ denote expectation under $P^{z}$. Let us also use the notation $E^{\emptyset}$ when the array starts empty, in which 
case at time $n$ only the portion $\left\{z_{k \ell}(n): 1 \leq k \leq N, 1 \leq \ell \leq k \wedge n\right\}$ of the array has been defined. Recall $y^{0, M}=\left(e^{-M \rho_{N, \ell}}\right)_{1 \leq \ell \leq N}$ with $\rho$ from (3.30).

Proposition 5.3. Let $N, n \geq 1$, and let $f$ be a bounded continuous function of the $(0, \infty)$-valued coordinates $\left\{z_{k \ell}(s): 1 \leq s \leq n, 1 \leq k \leq N, 1 \leq \ell \leq k \wedge s\right\}$. Then

$$
\lim _{M \rightarrow \infty} \int_{\mathbb{T}_{N}} \bar{K}_{\theta}^{N}\left(y^{0, M}, d z\right) E^{z}[f(z(1), \ldots, z(n))]=E^{\emptyset}[f(z(1), \ldots, z(n))] .
$$

Before turning to the proof of Proposition 5.3 we use it to derive part (iv) of Theorem 3.9. Let $\nu^{M}(d z)$ denote the initial distribution $\bar{K}_{\theta}^{N}\left(y^{0, M}, d z\right)$ on arrays, and let $f$ be a bounded continuous function on arrays. Then part (iv) follows from this calculation:

$$
\begin{aligned}
E^{\emptyset}[f(z(n))] & =\lim _{M \rightarrow \infty} E^{\nu^{M}}[f(z(n))]=\lim _{M \rightarrow \infty} E^{\nu^{M}} \int_{\mathbb{T}_{N}} \bar{K}_{\theta}^{N}(y(n), d z) f(z) \\
& =E^{\emptyset} \int_{\mathbb{T}_{N}} \bar{K}_{\theta}^{N}(y(n), d z) f(z)=\int_{\mathbb{Y}_{N}} \mu_{n}^{N}(d y) \int_{\mathbb{T}_{N}} \bar{K}_{\theta}^{N}(y, d z) f(z) .
\end{aligned}
$$

The first and third equalities are instances of (5.6), the second is (3.25), and the last one is Proposition 5.3 again because one consequence of limit (5.6), together with $\mu_{n}^{N}\left(y^{0, M}, d y\right) \rightarrow \mu_{n}^{N}(d y)$ from part (i), is that $\mu_{n}^{N}$ is the marginal distribution of $y(n)$ under $P^{\emptyset}$ for $n \geq N$. The third equality above is justified by arguing that $\int \bar{K}_{\theta}^{N}(y, d z) f(z)$ is a continuous function of $y$, or equivalently, that $y \mapsto \bar{K}_{\theta}^{N}(y, d z)$ is a continuous mapping into the space of probability measures on arrays (in the usual weak topology of probability measures, generated by bounded continuous functions). This follows from the fact that, off the bottom row, $\bar{K}_{\theta}^{N}(y, d z)$ has a density that is jointly continuous in $(y, z)$. Pointwise convergence of densities implies convergence of probability measures, a result known as Scheffé's theorem. This completes the proof of part (iv).

To prove part (v) of Theorem 3.9 we will use the alternative form of the Whittaker integral identity (3.21). By part (i) of the theorem, we have

$$
\int_{\mathbb{R}_{+}^{N}} f(y) \mu_{N}^{N}(d y)=\int_{\iota \mathbb{R}^{N}} d \lambda s_{N}(\lambda)\left(\int_{\mathbb{R}_{+}^{N}} f(y) \Psi_{\theta}^{N}(y) \Psi_{-\lambda}^{N}(y) \prod_{i} \frac{d y_{i}}{y_{i}}\right) \prod_{m=1}^{N} \prod_{i=1}^{N} \frac{\Gamma\left(\hat{\theta}_{m}+\lambda_{i}\right)}{\Gamma\left(\theta_{i}+\hat{\theta}_{m}\right)} .
$$

for any continuous, compactly supported function $f$ on $(0, \infty)^{N}$. By $(3.21)$

$$
\int_{(0, \infty)^{N}} e^{-y_{N}^{-1}} \Psi_{\lambda}^{N}(y) \Psi_{\hat{\theta}}^{N}(y) \prod_{i=1}^{N} \frac{d y_{i}}{y_{i}}=\prod_{m=1}^{N} \prod_{i=1}^{N} \Gamma\left(\lambda_{i}+\hat{\theta}_{m}\right) .
$$

As above, the functions $\int_{\mathbb{R}_{+}^{N}} f(y) \Psi_{\theta}^{N}(y) \Psi_{-\lambda}^{N}(y) \prod_{i} \frac{d y_{i}}{y_{i}}$ and $\prod_{m=1}^{N} \prod_{i=1}^{N} \Gamma\left(\lambda_{i}+\hat{\theta}_{m}\right)$ are both in $L^{2}\left(\iota \mathbb{R}^{N}, s_{N}(\lambda) d \lambda\right)$ so we have, by the Plancherel theorem,

$$
\int_{\mathbb{R}_{+}^{N}} f(y) \mu_{N}^{N}(d y)=\prod_{m=1}^{N} \prod_{i=1}^{N} \Gamma\left(\theta_{i}+\hat{\theta}_{m}\right)^{-1} \int_{\mathbb{R}_{+}^{N}} f(y) \Psi_{\theta}^{N}(y) e^{-y_{N}^{-1}} \Psi_{\hat{\theta}}^{N}(y) \prod_{i=1}^{N} \frac{d y_{i}}{y_{i}},
$$

as required.

Proof of Proposition 5.3. Figure 2 shows that $\left\{z_{k \ell}(s): 1 \leq s \leq n, 1 \leq k \leq N, 1 \leq \ell \leq k \wedge s\right\}$ can be written as a function of $\left\{z_{m+1}(m): 0 \leq m<n \wedge N\right\}$ and $d^{[1, n]}$. Let $(z(1), \ldots, z(n))=G\left(\left(z_{m+1}(m)\right)_{0 \leq m<n \wedge N}, d^{[1, n]}\right)$ represent this functional relationship defined by the row insertion procedure. The case of starting with an empty array is the one where each vector $z_{m+1}(m)=e_{1}^{(N-m)}$ where $e_{1}^{(k)}=(1,0, \ldots, 0)$ is the first $k$ dimensional unit vector. This can be seen from Figure 3. If we let $\mathbb{P}$ denote the probability distribution of the weight matrix $d$, the goal (5.6) can be re-expressed as

$$
\begin{gathered}
\lim _{M \rightarrow \infty} \int_{\mathbb{T}_{N}} \bar{K}_{\theta}^{N}\left(y^{0, M}, d z\right) \int_{\mathbb{R}_{+}^{n N}} \mathbb{P}\left(d\left(d^{[1, n]}\right)\right) f\left(G\left(\left(z_{m+1}(m)\right)_{0 \leq m<n \wedge N}, d^{[1, n]}\right)\right) \\
=\int_{\mathbb{R}_{+}^{n N}} \mathbb{P}\left(d\left(d^{[1, n]}\right)\right) f\left(G\left(\left(e_{1}^{(N-m)}\right)_{0 \leq m<n \wedge N}, d^{[1, n]}\right)\right) .
\end{gathered}
$$


(Notation $\mathbb{P}\left(d\left(d^{[1, n]}\right)\right)$ means that the matrix $d^{[1, n]}$ is the integration variable under the measure $\mathbb{P}$.) On the left above the vectors $\left(z_{m+1}(m)\right)_{0 \leq m<n \wedge N}$ are themselves functions of the initial values $\left(z_{m}(0)\right)_{0 \leq m<n \wedge N}=$ $\left(z_{m}\right)_{0 \leq m<n \wedge N}$ and the weights $d^{[1, n-1]}$, as shown in Figure 2. Comparison of the $\xi^{\prime}$ output in equations (2.1) and (2.2) shows that the mapping $G$ is continuous as the inputs $z_{m+1}(m) \rightarrow e_{1}^{(N-m)}$. Thus the upshot is that we need to show the weak convergence $\left(z_{m+1}(m)\right)_{0 \leq m<n \wedge N} \rightarrow\left(e_{1}^{(N-m)}\right)_{0 \leq m<n \wedge N}$ as $M \rightarrow \infty$. Since the limit is deterministic we can ignore the joint distribution and do this one coordinate at a time. So it suffices to fix $0 \leq m<k \leq N$ such that $m<n \wedge N$ and show that

$$
z_{k, m+1}(m) \rightarrow \delta_{k, m+1} \quad \text { in probability as } M \rightarrow \infty
$$

when $z_{k, m+1}(m)$ has the probability distribution described by the left-hand side of (5.7) and $\delta_{k, m+1}$ is the Kronecker delta.

Write

$$
z_{k, m+1}(m)=V_{k, m}\left[\left(z_{k, \ell}(0)\right)_{1 \leq \ell \leq m+1, \ell \leq k \leq N}, d^{[1, m]}\right]
$$

to indicate the functional relationship from the inputs to the array element $z_{k, m+1}(m)$. Our goal (5.8) follows if we show that for any fixed $d^{[1, m]} \in(0, \infty)^{m N}$ and a bounded continuous test function $f$,

$$
\lim _{M \rightarrow \infty} \int_{\mathbb{T}_{N}} \bar{K}_{\theta}^{N}\left(y^{0, M}, d z\right) f\left(V_{k, m}\left[\left(z_{k, \ell}\right)_{1 \leq \ell \leq m+1, \ell \leq k \leq N}, d^{[1, m]}\right]\right)=f\left(\delta_{k, m+1}\right) .
$$

To understand the asymptotics of $\bar{K}_{\theta}^{N}\left(y^{0, M}, d z\right)$ it is convenient to switch from multiplicative to additive variables. Define an array $t=\left\{t_{k \ell}\right\}_{1 \leq \ell \leq k \leq N}$ by $z_{k \ell}=e^{t_{k \ell}}$. Let $\widetilde{K}(y, d t)$ denote the distribution of the array $t$ when $z$ has distribution $\bar{K}_{\theta}^{N}(y, d z)$. For $u \in \mathbb{R}^{N}$ let

$$
W(u)=\left\{t=\left\{t_{k \ell}\right\}_{1 \leq \ell \leq k \leq N}: t_{N, i}=u_{i}, 1 \leq i \leq N\right\}
$$

be the set of arrays with bottom row $u$. Let

$$
\mathcal{F}_{\theta}(t)=\sum_{k=1}^{N} \theta_{k}\left(\sum_{\ell=1}^{k-1} t_{k-1, \ell}-\sum_{\ell=1}^{k} t_{k, \ell}\right)-\sum_{k=1}^{N-1} \sum_{\ell=1}^{k}\left(e^{t_{k, \ell}-t_{k+1, \ell}}+e^{t_{k+1, \ell+1}-t_{k, \ell}}\right) .
$$

Then, for a bounded continuous test function $g$,

$$
\begin{aligned}
\int g(t) \widetilde{K}\left(y^{0, M}, d t\right) & =\frac{1}{C(M)} \int_{W\left(-M \rho^{[n]}\right)} g(t) e^{\mathcal{F}_{\theta}(t)} d t \\
& =\frac{1}{C(M)} \int_{W(0)} g(t-M \rho) e^{S_{\theta}(t)+e^{M / 2} \mathcal{F}_{0}(t)} d t
\end{aligned}
$$

Above $C(M)=\int_{W(0)} e^{S_{\theta}(t)+e^{M / 2} \mathcal{F}_{0}(t)} d t$ is the normalization needed for a probability measure. We changed variables by shifting $t$ to $t-M \rho$ where $\rho=\left(\rho_{k \ell}\right)_{1 \leq \ell \leq k \leq N}$ is the array from (3.30) defined by $\rho_{k \ell}=\frac{1}{2}(k-$ $1)-\ell+1$. Defining $S_{\theta}(t)=\mathcal{F}_{\theta}(t)-\mathcal{F}_{0}(t)$ leads to

$$
\mathcal{F}_{\theta}(t-M \rho)=S_{\theta}(t-M \rho)+\mathcal{F}_{0}(t-M \rho)=S_{\theta}(t)+e^{M / 2} \mathcal{F}_{0}(t) .
$$

Return to the right-hand side of (5.10) to rewrite as

$$
\begin{aligned}
& \int_{\mathbb{T}_{N}} \bar{K}_{\theta}^{N}\left(y^{0, M}, d z\right) f\left(V_{k, m}\left[\left(z_{k, \ell}\right)_{1 \leq \ell \leq m+1, \ell \leq k \leq N}, d^{[1, m]}\right]\right) \\
& =\int \widetilde{K}\left(y^{0, M}, d t\right) f\left(V_{k, m}\left[\left(e^{t_{k \ell}}\right)_{1 \leq \ell \leq m+1, \ell \leq k \leq N}, d^{[1, m]}\right]\right) \\
& =\frac{1}{C(M)} \int_{W(0)} f\left(V_{k, m}\left[\left(e^{t_{k \ell}-M \rho_{k \ell}}\right)_{1 \leq \ell \leq m+1, \ell \leq k \leq N}, d^{[1, m]}\right]\right) e^{S_{\theta}(t)+e^{M / 2} \mathcal{F}_{0}(t)} d t .
\end{aligned}
$$

We claim that

$$
\text { line (5.13) converges to } f\left(\delta_{k, m+1}\right) \text { as } M \rightarrow \infty \text {. }
$$

Limit (5.14) finishes the proof of Proposition 5.3. To establish it we prove the two lemmas below. 
Lemma 5.4. On the set $W(0)$, the function $\mathcal{F}_{0}$ is strictly concave and has a unique maximum $t^{0}$ that satisfies $\sum_{\ell=1}^{k} t_{k \ell}^{0}=0$ for each $1 \leq k \leq N$.

Lemma 5.5. For each fixed $1 \leq m+1 \leq k \leq N, m<n, d^{[1, m]} \in(0, \infty)^{m N}$,

$$
\lim _{\substack{M \rightarrow \infty \\ t \rightarrow t^{0}}} z_{k, m+1}(m)=\lim _{\substack{M \rightarrow \infty \\ t \rightarrow t^{0}}} V_{k, m}\left[\left(e^{t_{k \ell}-M \rho_{k \ell}}\right)_{1 \leq \ell \leq m+1, \ell \leq k \leq N}, d^{[1, m]}\right]=\delta_{k, m+1} .
$$

Lemma 5.4 implies that the probability measure $C(M)^{-1} \mathbf{1}_{W(0)}(t) e^{S_{\theta}(t)+e^{M / 2} \mathcal{F}_{0}(t)} d t$ converges weakly to the pointmass at $t^{0}$. This together with (5.15) and the boundedness and continuity of $f$ imply (5.14). We have proved Proposition 5.3 but it remains to prove the lemmas above.

Proof of Lemma 5.4. This lemma comes from [44] and [53]. We include the proof for the sake of completeness.

The critical point equations $\frac{\partial}{\partial t_{k i}} \mathcal{F}_{0}(t)=0$ for $1 \leq i \leq k<N$ rearrange to

$$
e^{2 t_{k i}}=\frac{e^{t_{k-1, i}} \mathbf{1}_{\{i<k\}}+e^{t_{k+1, i+1}}}{e^{-t_{k-1, i-1}} \mathbf{1}_{\{k \geq i>1\}}+e^{-t_{k+1, i}}} \quad \text { for } 1 \leq i \leq k<N .
$$

The case $k=1$ gives $t_{11}=\left(t_{21}+t_{22}\right) / 2$. Multiplying equations together gives

$$
e^{2 \sum_{i=1}^{k} t_{k i}}=e^{\sum_{i=1}^{k-1} t_{k-1, i}+\sum_{i=1}^{k+1} t_{k+1, i+1}} \quad \text { for } 2 \leq k \leq N .
$$

From this follows $k^{-1} \sum_{i=1}^{k} t_{k i}=N^{-1} \sum_{i=1}^{k} t_{N i}$ for $1 \leq k<N$, and these all $=0$ by the $W(0)$ condition.

Following [53, p. 136] we write $\mathcal{F}_{0}$ in the following form. Consider the directed graph $(\mathcal{V}, \mathcal{E})$ with vertex set $\mathcal{V}=\{(k, i): 1 \leq i \leq k \leq N\}$ and where $\mathcal{E}$ contains all possible edges $((k+1, i),(k, i))$ and $((k, i),(k+1, i+1))$. Edge $a=(u(a), v(a))$ is directed form vertex $u(a)$ to vertex $v(a)$. Then

$$
\mathcal{F}_{0}(t)=-\sum_{a \in \mathcal{E}} e^{t_{v(a)}-t_{u(a)}}
$$

and for vertices $x, y$

$$
\frac{\partial^{2} \mathcal{F}_{0}(t)}{\partial t_{x} \partial t_{y}}=-\left(\sum_{a \in \mathcal{E}: v(a)=x} e^{t_{x}-t_{u(a)}}+\sum_{a \in \mathcal{E}: u(a)=x} e^{t_{v(a)}-t_{x}}\right) \mathbf{1}_{\{x=y\}}+e^{t_{x}-t_{y}} \mathbf{1}_{\{(y, x) \in \mathcal{E}\}}+e^{t_{y}-t_{x}} \mathbf{1}_{\{(x, y) \in \mathcal{E}\}}
$$

Take a vector $\left(\alpha_{x}\right)_{x \in \mathcal{V}}$ such that $\alpha_{(N, i)}=0$ (because the variables $t_{N, i}$ are not free to vary on $\left.W(0)\right)$. Then

$$
\sum_{x, y \in \mathcal{V}} \alpha_{x} \alpha_{y} \frac{\partial^{2} \mathcal{F}_{0}(t)}{\partial t_{x} \partial t_{y}}=-\sum_{a \in \mathcal{E}}\left(\alpha_{v(a)}-\alpha_{u(a)}\right)^{2} e^{t_{v(a)}-t_{u(a)}}
$$

is $<0$ unless $\alpha=0$. This gives strict concavity and the unique maximum.

Proof of Lemma 5.5. First we take care of the case $m=0$. This is read off directly from the initial values and $t_{11}^{0}=0: z_{k, 1}(0)=e^{t_{k, 1}+M(1-(k+1) / 2)} \rightarrow \delta_{k, 1}$ as $M \rightarrow \infty$ and $t \rightarrow t^{0}$.

For the rest of the proof $m \geq 1$. We turn to the matrix machinery developed in [43]. For that purpose we consider the row insertion procedure also in terms of ratio variables. Let $\eta=\left(\eta_{\ell}, \ldots, \eta_{N}\right)$ denote the ratio variables associated with the vector $\xi=\left(\xi_{\ell}, \ldots, \xi_{N}\right): \xi_{k}=\eta_{\ell} \eta_{\ell+1} \cdots \eta_{k}$ for $k=\ell, \ldots, N$. Similarly $\xi_{k}^{\prime}=\eta_{\ell}^{\prime} \eta_{\ell+1}^{\prime} \cdots \eta_{k}^{\prime}$. Then the row insertion

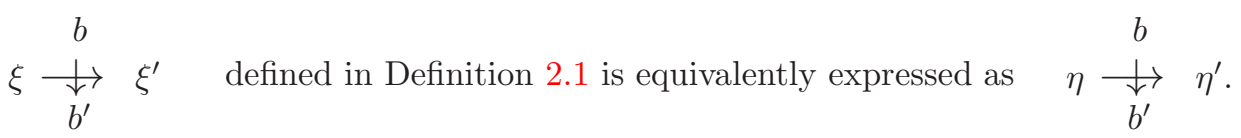

Recall definition (2.13) of the $N \times N$ matrices $H_{m}(\eta)$. Then (5.16) is equivalent to [43, eqn. (2.23)-(2.25)]

$$
H_{\ell}(\eta) H_{\ell}(b)=H_{\ell+1}\left(b^{\prime}\right) H_{\ell}\left(\eta^{\prime}\right) \text {. }
$$

In the extreme case $\ell=N$ there is no $b^{\prime}$ left and the correct interpretation is $H_{N+1}\left(b^{\prime}\right)=I=$ the $N \times N$ identity matrix.

As in the end of Section 2, define the ratio variables of the arrays by

$$
\eta_{\ell \ell}(n)=z_{\ell \ell}(n) \text { and } \eta_{k \ell}(n)=z_{k \ell}(n) / z_{k-1, \ell}(n) \text { for } 1 \leq \ell<k .
$$




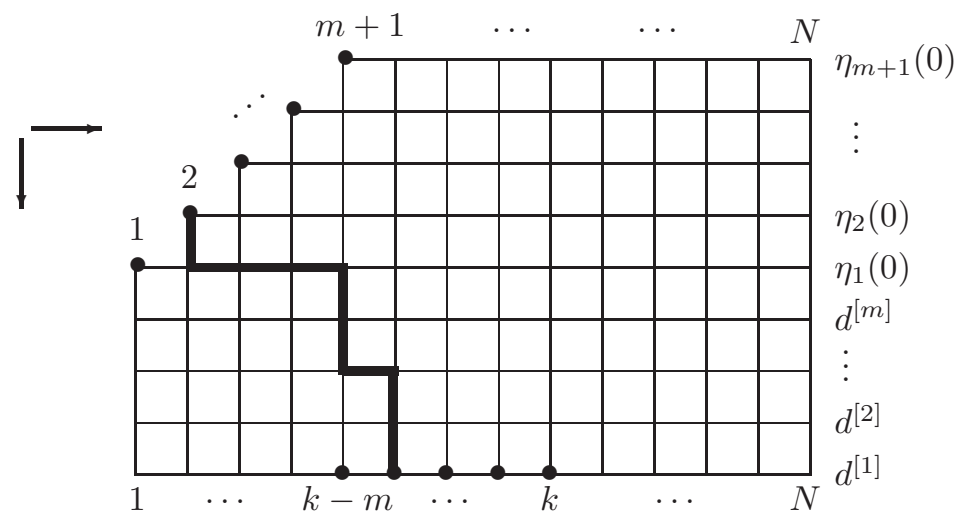

Figure 5. The minor $\tau_{k}^{m+1}$ equals the sum of the weights of $(m+1)$-tuples of disjoint down-right paths $\left(\gamma^{1}, \ldots, \gamma^{m+1}\right)$, where $\gamma^{j}$ is a path from vertex $j$ at the top to vertex $k-m-1+j$ at the bottom. The thickset line displays one admissible path $\gamma^{2}$ from top vertex 2 to bottom vertex $k-m+1$.

Applying (5.17) to the upper left corner of Figure 2 gives

$$
H_{1}\left(\eta_{1}(0)\right) H_{1}\left(a_{1}(1)\right)=H_{2}\left(a_{2}(1)\right) H_{1}\left(\eta_{1}(1)\right) .
$$

Left multiply this identity by $H_{2}\left(\eta_{2}(0)\right), H_{3}\left(\eta_{3}(0)\right), \ldots$, right multiply by $H_{1}\left(a_{1}(2)\right), H_{1}\left(a_{1}(3)\right), \ldots$, and apply (5.17) repeatedly on the right-hand side. This gives the following identity for all $m \geq 1$ :

$$
\prod_{i=0}^{m} H_{m+1-i}\left(\eta_{m+1-i}(0)\right) \cdot \prod_{j=1}^{m} H_{1}\left(a_{1}(j)\right)=\prod_{j=1}^{m} H_{m+2}\left(a_{m+2}(j)\right) \cdot \prod_{i=0}^{m} H_{m+1-i}\left(\eta_{m+1-i}(m)\right)
$$

If $m=N-1$ then $H_{m+2}\left(a_{m+2}(j)\right)=I$ and the first product on the right disappears. If $m<N-1$ then apply [43, Thm. 2.4] to the lower right $(N-m-1) \times(N-m-1)$ block of the first product on the right. This gives vectors $p^{m+\ell+1}, \ldots, p^{m+2}$ such that $p^{i}=\left(p_{i}^{i}, \ldots, p_{N}^{i}\right)$,

$$
\prod_{j=1}^{m} H_{m+2}\left(a_{m+2}(j)\right)=\prod_{j=0}^{\ell-1} H_{m+\ell+1-j}\left(p^{m+\ell+1-j}\right),
$$

and $\ell=m \wedge(N-m-1)$. Substituting this back into (5.19) gives

$$
\prod_{i=0}^{m} H_{m+1-i}\left(\eta_{m+1-i}(0)\right) \cdot \prod_{j=1}^{m} H_{1}\left(d^{[j]}\right)=\prod_{j=0}^{\ell-1} H_{m+\ell+1-j}\left(p^{m+\ell+1-j}\right) \cdot \prod_{i=0}^{m} H_{m+1-i}\left(\eta_{m+1-i}(m)\right) .
$$

Let $H$ denote the matrix on the left. On the right we have a descending sequence of subscripts $(2 m+1) \wedge$ $N, \ldots, 1$. We can appeal to [43, Prop. 1.6] to conclude that the vectors on the right-hand side are uniquely determined. In particular, $\eta_{m+1}(m)=\left(\eta_{k, m+1}(m)\right)_{k=m+1}^{N}$ is given by

$$
\eta_{m+1, m+1}(m)=\frac{\tau_{m+1}^{m+1}}{\tau_{m+1}^{m}}, \quad \eta_{k, m+1}(m)=\frac{\tau_{k}^{m+1} \tau_{k-1}^{m}}{\tau_{k}^{m} \tau_{k-1}^{m+1}} \quad \text { for } m+1<k \leq N,
$$

where $\tau_{j}^{i}=\operatorname{det} H_{[j-i+1, j]}^{[1, i]}, i \leq j$, are minor determinants of the matrix $H$ over rows $1, \ldots, i$ and columns $j-i+1, \ldots, j$. Switching back to $z$ via (5.18) gives

$$
z_{k, m+1}(m)=\frac{\tau_{k}^{m+1}}{\tau_{k}^{m}} \quad \text { for } m+1 \leq k \leq N
$$

This is the function $V_{k, m}$ defined in (5.9). (Prop. 1.6 of [43] needs a hypothesis on the minors of $H$. This hypothesis can be checked from (5.23) below with the help of Figure 5.)

We use a graphical representation to compute the minors $\tau_{j}^{i}$, in the spirit of the Lindström-Gessel-Viennot method, following Sect. 1.1 of [43]. The matrix $H$ is represented by an array of $2 m+1$ right-adjusted rows, one row for each vector $\eta_{m+1}(0), \ldots, \eta_{1}(0), d^{[1]}, \ldots, d^{[m]}$ (see Figure 5). For $1 \leq i \leq m+1$, the vertices on row $i$ are assigned weights $\eta_{m+2-i, m+2-i}(0), \ldots, \eta_{N, m+2-i}(0)$, and for $m+2 \leq i \leq 2 m+1$, the vertices 


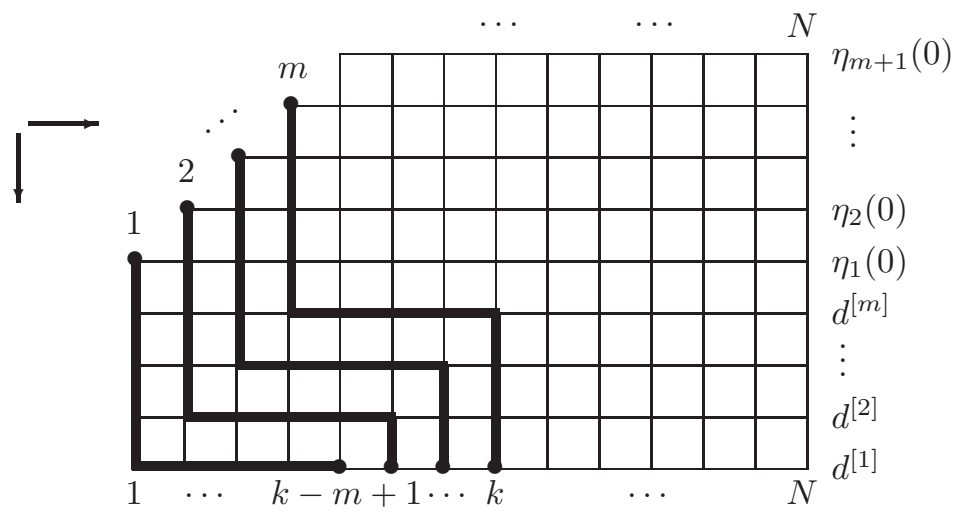

FiguRE 6. An $m$-tuple of paths for the minor $\tau_{k}^{m}$.

on row $i$ are assigned weights $d_{i-m-1,1}, \ldots, d_{i-m-1, N}$. Note that due to initial elements missing from the $\eta$-vectors, the top vertex of column $j$ is on row $m-j+2$ for $1 \leq j \leq m$. Combining (1.16) and (1.27) in [43] gives

$$
\tau_{j}^{i}=\operatorname{det} H_{[j-i+1, j]}^{[1, i]}=\sum_{\left(\gamma^{1}, \ldots, \gamma^{i}\right)} w t\left(\gamma^{1}, \ldots, \gamma^{i}\right)
$$

where the sum ranges over $i$-tuples of disjoint paths $\gamma^{1}, \ldots, \gamma^{i}$ such that $\gamma^{k}$ goes from vertex $k$ at the top edge of the graph to vertex $j-i+k$ at the bottom edge, and the weight $w t\left(\gamma^{1}, \ldots, \gamma^{i}\right)$ of the $i$-tuple is the product of the weights on the vertices of the paths.

Now we find the asymptotics of the minors in (5.22). We are proving (5.15) so the initial $z_{k \ell}$-values are $z_{k \ell}=e^{t_{k \ell}-M \rho_{k \ell}}=e^{t_{k \ell}+M(\ell-(k+1) / 2)}$. From this we get the initial ratio variables

$$
\eta_{\ell \ell}=z_{\ell \ell}=e^{t_{\ell \ell}+M(\ell-1) / 2} \text {, and } \eta_{k \ell}=z_{k \ell} / z_{k-1, \ell}=e^{t_{k \ell}-t_{k-1, \ell}-M / 2} \text { for } k>\ell .
$$

Note in particular that on the top $m+1$ rows of the array in Figure 5, all but the left edge weights decay as $C e^{-M / 2}$.

Consider first $\tau_{k}^{m+1}$ for some $k>m+1$. One can check by induction on $m$ that every $(m+1)$-tuple of paths $\left(\gamma^{1}, \ldots, \gamma^{m+1}\right)$ from vertices $(1, \ldots, m+1)$ on the top edge to vertices $(k-m, \ldots, k)$ on the bottom edge contains at least $m(m+1) / 2+1$ vertices with weight $\eta_{k \ell}$ with $\ell<k$. Since the $t_{k \ell}$ variables converge to a finite constant, up to a constant multiple

$$
w t\left(\gamma^{1}, \ldots, \gamma^{m+1}\right) \leq C \prod_{\ell=1}^{m+1} e^{M(\ell-1) / 2} \cdot\left(e^{-M / 2}\right)^{m(m+1) / 2+1} \leq C e^{-M / 2} .
$$

There is a fixed finite number of these $(m+1)$-tuples in (5.23), and so $\tau_{k}^{m+1} \leq C e^{-M / 2}$ for $k>m+1$.

Next we establish a lower bound for $\tau_{k}^{m}$. The $m$ rows of $d$-weights at the bottom of the array allow an $m$-tuple of paths that uses exactly $m(m-1) / 2$ vertices with weight $\eta_{k \ell}$ with $\ell<k$ (Figure 6 ). This $m$-tuple gives a positive constant lower bound: $\tau_{k}^{m} \geq c>0$.

Combination of the first two bounds gives

$$
z_{k, m+1}(m)=\frac{\tau_{k}^{m+1}}{\tau_{k}^{m}} \leq C e^{-M / 2} \rightarrow 0 \quad \text { for } m+1<k \leq N .
$$

It remains to consider the case $k=m+1$. Minor $\tau_{m+1}^{m+1}$ has a unique admissible $(m+1)$-tuple, namely $m+1$ vertical paths. Consequently

$$
\tau_{m+1}^{m+1}=\prod_{1 \leq \ell \leq k \leq m+1} \eta_{k \ell} \cdot \prod_{\substack{1 \leq i \leq m \\ 1 \leq j \leq m+1}} d_{i, j}=e^{\sum_{\ell=1}^{m+1} t_{m+1, \ell}} \cdot \prod_{\substack{1 \leq i \leq m \\ 1 \leq j \leq m+1}} d_{i, j} .
$$


For $\tau_{m+1}^{m}$ the $m$-tuple in Figure 6 has weight

$$
\prod_{1 \leq \ell \leq k \leq m} \eta_{k \ell} \cdot \prod_{\substack{1 \leq i \leq m \\ 1 \leq j \leq m+1}} d_{i, j}=e^{\sum_{\ell=1}^{m} t_{m, \ell}} \cdot \prod_{\substack{1 \leq i \leq m \\ 1 \leq j \leq m+1}} d_{i, j} .
$$

This $m$-tuple is minimal in its use of weights $\eta_{k \ell}$ with $\ell<k$. Other admissible $m$-tuples for $\tau_{m+1}^{m}$ necessarily use more of such weights and consequently pick up more $e^{-M / 2}$ factors. From all this

$$
\begin{gathered}
z_{m+1, m+1}(m)=\frac{\tau_{m+1}^{m+1}}{\tau_{m+1}^{m}}=\frac{e^{\sum_{\ell=1}^{m+1} t_{m+1, \ell}} \cdot \prod_{\substack{1 \leq i \leq m \\
1 \leq j \leq m+1}} d_{i, j}}{e^{\sum_{\ell=1}^{m} t_{m, \ell}} \cdot \prod_{\substack{1 \leq i \leq m \\
1 \leq j \leq m+1}} d_{i, j}+O\left(e^{-M / 2}\right)} \\
\underset{M \rightarrow \infty, t \rightarrow t^{0}}{\longrightarrow} \frac{e^{\sum_{\ell=1}^{m+1} t_{m+1, \ell}^{0}}}{e^{\sum_{\ell=1}^{m} t_{m, \ell}^{0}}}=\frac{e^{0}}{e^{0}}=1 .
\end{gathered}
$$

(5.24) and (5.25) together verify (5.15) and complete the proof of Lemma 5.5.

\section{REFERENCES}

[1] M. Adler, P. van Moerbeke. PDEs for the joint distributions of the Dyson, Airy and Sine processes. Ann. Probab., 33:1326$1361(2005)$.

[2] D. Aldous, P. Diaconis. Longest increasing subsequences: From patience sorting to the Baik-Deift-Johansson theorem. Bull. Amer. Math. Soc., 36: 413-432 (1999).

[3] J. Baik, P.A. Deift, K. Johansson. On the distribution of the length of the longest increasing subsequence of random permutations. J. Amer. Math. Soc., 12:1119-1178 (1999).

[4] M. Balázs, E. Cator, T. Seppäläinen. Cube root fluctuations for the corner growth model associated to the exclusion process. Electron. J. Probab., 11:1094-1132 (2006).

[5] M. Balázs, T. Seppäläinen. Order of current variance and diffusivity in the asymmetric simple exclusion process. Ann. of Math., 171:1237-1265 (2010).

[6] F. Baudoin, N. O'Connell. Exponential functionals of Brownian motion and class one Whittaker functions. Ann. Inst. H. Poincaré $B$, in press.

[7] A. Berenstein, D. Kazhdan. Geometric and unipotent crystals. Visions in Mathematics, Modern Birkhäuser Classics, 188$236,2010$.

[8] A. Berenstein, D. Kazhdan. Lecture notes on geometric crystals and their combinatorial analogues. Combinatorial aspect of integrable systems, MSJ Memoirs, 17, Mathematical Society of Japan, Tokyo, 2007

[9] A. Berenstein, A.N. Kirillov. The Robinson-Schensted-Knuth bijection, quantum matrices and piece-wise linear combinatorics. Proceedings of 13th International Conference on Formal Power Series and Algebraic Combinatorics, Arizona State University, May 20-26, 2001.

[10] Ph. Biane, Ph. Bougerol, N. O'Connell. Littelmann paths and Brownian paths. Duke Math. J., 130:127-167 (2005).

[11] Ph. Biane, Ph. Bougerol, N. O'Connell. Continuous crystals and Duistermaat-Heckman measure for Coxeter groups. Adv. Math., 221:1522-1583 (2009).

[12] A. Borodin, I. Corwin Macdonald processes. Probab. Th. Rel. Fields, to appear. arXiv:1111.4408.

[13] A. Borodin, I. Corwin, D. Remenik. Log-Gamma polymer free energy fluctuations via a Fredholm determinant identity. Commun. Pure Appl. Math., to appear. arXiv:1206.4573.

[14] A. Borodin, S. Péché. Airy kernel with two sets of parameters in directed percolation and random matrix theory. J. Stat. Phys., 132:275-290 (2008).

[15] Ph. Bougerol, Th. Jeulin. Paths in Weyl chambers and random matrices. Probab. Th. Rel. Fields, 124:517-543 (2002).

[16] D. Bump. Automorphic forms on $G L(3, \mathbb{R})$. Lecture Notes in Mathematics, 1083. Springer-Verlag, Berlin, 1984.

[17] D. Bump. The Rankin-Selberg method: a survey, in Number Theory, Trace Formulas, and Discrete Groups (K. E. Aubert,

E. Bombieri and D. Goldfeld, eds.). Academic Press, New York, 1989.

[18] E. Cator, P. Groeneboom. Second class particles and cube root asymptotics for Hammersley's process Ann. Probab., 34:1273-1295, (2006).

[19] I. Corwin. The Kardar-Parisi-Zhang equation and universality class. Random Matrices: Theory and Appl. 1:1130001 (2012)

[20] I. Corwin, A. Hammond. The $H$-Brownian Gibbs property of the KPZ line ensemble. In preparation.

[21] V. Danilov, G. Koshevoy. The octahedron recurrence and RSK-correspondence. Seminar Lotharinngien de Combinatoire, B54An (2007).

[22] M. Defosseux. Orbit measures, random matrix theory and interlaced determinantal processes. Ann. Inst. H. Poincaré B, 46:209-249 (2010).

[23] A.B. Dieker, J. Warren. On the largest-eigenvalue process for generalized Wishart random matrices ALEA, 6:369-376 (2009).

[24] Y. Doumerc, A note on representations of eigenvalues of classical Gaussian matrices. Sèminaire de Probabilités XXXVII. Lecture Notes in Math., 1832:370-384 (2003).

[25] M. Draief, J. Mairesse, N. O'Connell. Queues, Stores, and Tableaux. J. Appl. Probab., 42:1145-1167 (2005). 
[26] P.L. Ferrari, H. Spohn. Random growth models. arXiv:1003.0881.

[27] P. J. Forrester, E. M. Rains. Jacobians and rank 1 perturbations relating to unitary Hessenberg matrices. Int. Math. Res. Not., 48306 (2006).

[28] A. Gerasimov, S. Kharchev, D. Lebedev, S. Oblezin. On a Gauss-Givental representation of quantum Toda chain wave equation. Int. Math. Res. Notices 1-23 (2006).

[29] A. Gerasimov, D. Lebedev, S. Oblezin. Baxter Operator and Archimedean Hecke Algebra. Commun. Math. Phys. 284:867896 (2008).

[30] A. Givental. Stationary phase integrals, quantum Toda lattices, flag manifolds and the mirror conjecture. Topics in Singularity Theory, AMS Transl. Ser. 2, vol. 180, AMS, Rhode Island (1997) 103-115.

[31] D. Goldfeld, A. Kontorovich. On the determination of the Plancherel measure for Lebedev-Whittaker transforms on $G L(n)$. arXiv: 1102.5086

[32] C. Greene. An extension of Schensted's theorem. Adv. Math. 14:254-265 (1974).

[33] K. Johansson. Shape fluctuations and random matrices. Comm. Math. Phys., 209:437-476 (2000).

[34] K. Johansson. Discrete orthogonal polynomial ensembles and the Plancherel measure Ann. of Math., 153:259-296 (2001).

[35] S. Kharchev, D. Lebedev. Integral representations for the eigenfunctions of quantum open and periodic Toda chains from the QISM formalism. J. Phys. A, 34:2247-2258 (2001).

[36] A. N. Kirillov. Introduction to tropical combinatorics. In: Physics and Combinatorics. Proc. Nagoya 2000 2nd Internat. Workshop (A. N. Kirillov and N. Liskova, eds.), World Scientific, Singapore, 82-150, 2001,

[37] W. König, N. O'Connell, S. Roch. Non-colliding random walks, tandem queues, and discrete orthogonal polynomial ensembles. Electron. J. Probab. 7 (2002)

[38] B. Kostant. Quantisation and representation theory. In: Representation Theory of Lie Groups, Proc. SRC/LMS Research Symposium, Oxford 1977, LMS Lecture Notes 34, Cambridge University Press, 1977, pp. 287-316.

[39] B. F. Logan, L. A. Shepp. A variational problem for random Young tableaux. Adv. Math., 26:206-222 (1977).

[40] I. Macdonald. Symmetric Functions and Hall Polynomials. Second Edition, Oxford University Press, 1995.

[41] H. Matsumoto, M. Yor. A version of Pitman's $2 M-X$ theorem for geometric Brownian motions. C. R. Acad. Sci. Paris 328:1067-1074 (1999).

[42] J. Moriarty, N. O'Connell. On the free energy of a directed polymer in a Brownian environment. Markov Process. Rel. Fields 13:251-266 (2007).

[43] M. Noumi, Y. Yamada. Tropical Robinson-Schensted-Knuth correspondence and birational Weyl group actions. Representation theory of algebraic groups and quantum groups, 371-442, Adv. Stud. Pure Math., 40, Math. Soc. Japan, Tokyo, 2004 .

[44] N. O'Connell. Directed polymers and the quantum Toda lattice Ann. Probab., 40:437-458 (2012).

[45] N. O'Connell. Conditioned random walks and the RSK correspondence. J. Phys. A, 36:3049-3066 (2003).

[46] N. O'Connell. A path-transformation for random walks and the Robinson-Schensted correspondence. Trans. Amer. Math. Soc., 355:3669-3697 (2003).

[47] N. O'Connell. Random matrices, non-colliding processes and queues. Seminaire de Probabilites XXXVI, 165-182. Lecture Notes in Mathematics 1801, Springer, 2002.

[48] N. O'Connell, T. Seppäläinen, N. Zygouras. Geometric RSK correspondence, Whittaker functions and symmetrized random polymers. arXiv:1210.5126.

[49] N. O'Connell, J. Warren. A multi-layer extension of the stochastic heat equation. arXiv:1104.3509.

[50] N. O'Connell, M. Yor. Brownian analogues of Burke's theorem. Stochastic Process. Appl., 96:285-304 (2001).

[51] N. O'Connell, M. Yor. A representation for non-colliding random walks. Electron. Comm. Probab., 7 (2002).

[52] A. Okounkov. Infinite wedge and random partitions Selecta Math.,7:57-81 (2001).

[53] K. Rietsch. A mirror construction for the totally nonnegative part of the Peterson variety. Nagoya Math. J. 183:105-142 (2006).

[54] L.C.G. Rogers, J.W. Pitman. Markov functions. Ann. Probab., 9:573-582 (1981).

[55] T. Sasamoto, H. Spohn. The $1+1$-dimensional Kardar-Parisi-Zhang equation and its universality class. Proceedings StatPhys 24; Journal of Statistical Mechanics, online (2010).

[56] M. Semenov-Tian-Shansky. Quantisation of open Toda lattices. In: Dynamical systems VII: Integrable systems, nonholonomic dynamical systems. Edited by V. I. Arnol'd and S. P. Novikov. Encyclopaedia of Mathematical Sciences, 16. Springer-Verlag, 1994.

[57] T. Seppäläinen. Hydrodynamic scaling, convex duality and asymptotic shapes of growth models. Markov Process. Rel. Fields, 4:1-26 (1998).

[58] T. Seppäläinen. Exact limiting shape for a simplified model of first-passage percolation on the plane. Ann. Probab., 26:1232-1250 (1999).

[59] T. Seppäläinen. Scaling for a one-dimensional directed polymer with boundary conditions. Ann. Probab., 40:19-73 (2012).

[60] T. Seppäläinen, B. Valko. Bounds for scaling exponents for a $1+1$ dimensional directed polymer in a Brownian environment. ALEA Lat. Am. J. Probab. Math. Stat., 7:451-476 (2010).

[61] E. Stade. Archimedean $L$-factors on $G L(n) \times G L(n)$ and generalized Barnes integrals. Israel J. Math. 127:201-219 (2002).

[62] C. Tracy and H. Widom. Level-spacing distributions and the Airy kernel. Comm. Math. Phys., 159:151-174 (1994).

[63] A. M. Vershik, S. Kerov. Asymptotics of the Plancherel measure of the symmetric group and the limiting form of Young tables. Soviet Math. Dokl., 18:527-531 (1977). 
I. Corwin, Massachusetts Institute of Technology, Department of Mathematics, 77 Massachusetts Avenue, Cambridge, MA 02139-4307, USA

Clay Mathematics Institute, 10 Memorial Blvd. Suite 902, Providence, Ri 02903, USA

E-mail address: ivan.corwin@gmail.com

N. O'Connell, Mathematics Institute, University of Warwick, Coventry CV4 7AL, UK

E-mail address: N.M.0-Connell@warwick.ac.uk

T. Seppäläinen, Department of Mathematics, University of Wisconsin-Madison, 419 Van Vleck Hall, Madison, WI 53706-1388, USA

E-mail address: seppalai@math.wisc.edu

N. Zygouras, Department of Statistics, University of Warwick, Coventry CV4 7AL, UK

E-mail address: N.Zygouras@warwick.ac.uk 